\title{
Nonlinear shell models of Kirchhoff-Love type: existence theorem and comparison with Koiter's model
}

\author{
Cristinel Mardare
}

December 21, 2018

\author{
Sorbonne Université, CNRS, Laboratoire Jacques-Louis Lions, LJLL, \\ 75252 Paris cedex 05, France \\ E-mail: mardare@ann.jussieu.fr
}

Dedicated to Professor Philippe G. Ciarlet on the occasion of its 80th birthday

\begin{abstract}
We define two nonlinear shell models whereby the deformation of an elastic shell with small thickness minimizes ad hoc functionals over sets of admissible deformations of Kirchhoff-Love type. We establish that both models are close in a specific sense to the well-known nonlinear shell model of W.T. Koiter and that one of them has a solution, by contrast with Koiter's model for which such an existence theorem is yet to be proven.
\end{abstract}

2000 Mathematical Subject Classification: 74B20, 74K25, 35A35, 35B40

Keywords: Nonlinearly Elastic Shells; Kirchhoff-Love, Koiter, Existence Theory, Asymptotics

\section{Introduction}

A shell is an elastic body in the three-dimensional Euclidean space that lies within a thin tubular neighbourhood of a surface, hereafter referred to as the "middle surface of the shell". The objective of this paper is to introduce a wellposed minimization problem that predicts the deformation of shells in reponse to applied forces independent of time. It is valid under the following set of assumptions.

We assume that the elastic material constituting the shell is homogeneous, isotropic and frame-indifferent, so that its constitutive law in a stress-free reference configuration of the shell is governed by two Lamé constants

$$
\lambda>0 \text { and } \mu>0 .
$$


We assume that the middle surface of a stress-free reference configuration of the shell is a surface with boundary defined by

$$
S:=\boldsymbol{\theta}(\bar{\omega}) \subset \mathbb{E}^{3},
$$

where

$$
\omega \subset \mathbb{R}^{2}
$$

is a bounded and connected open subset of $\mathbb{R}^{2}$ with a Lipschitz-continuous boundary (in the sense of [16]), and where

$$
\boldsymbol{\theta} \in \mathcal{C}^{1}\left(\bar{\omega} ; \mathbb{E}^{3}\right)
$$

is an immersion such that

$$
\boldsymbol{a}_{3}:=\frac{\partial_{1} \boldsymbol{\theta} \wedge \partial_{2} \boldsymbol{\theta}}{\left|\partial_{1} \boldsymbol{\theta} \wedge \partial_{2} \boldsymbol{\theta}\right|} \in \mathcal{C}^{1}\left(\bar{\omega} ; \mathbb{E}^{3}\right)
$$

(here, and in the sequel, $y=\left(y_{1}, y_{2}\right)$ denotes the Cartesian coordinates of a generic point in $\bar{\omega}$ and $\left.\partial_{\alpha}:=\partial / \partial y_{\alpha}\right)$.

The assumption that $\boldsymbol{\theta}$ is an immersion means that the two vector fields

$$
\boldsymbol{a}_{\alpha}:=\partial_{\alpha} \boldsymbol{\theta} \in \mathcal{C}^{0}\left(\bar{\omega} ; \mathbb{E}^{3}\right), \alpha=1,2,
$$

are linearly independent at each point of $\bar{\omega}$; hence, for each $y \in \bar{\omega}$, the vectors $\boldsymbol{a}_{1}(y) \in \mathbb{E}^{3}$ and $\boldsymbol{a}_{2}(y) \in \mathbb{E}^{3}$ form a basis in the tangent space to $S$ at the point $\boldsymbol{\theta}(y) \in S$, and the vector $\boldsymbol{a}_{3}(y)$ is unit and normal to $S$ at $\boldsymbol{\theta}(y)$ (i.e., $\left|\boldsymbol{a}_{3}(y)\right|=1$ and $\boldsymbol{a}_{3}(y) \cdot \boldsymbol{a}_{\alpha}(y)=0$ for all $\left.\alpha=1,2\right)$.

We assume that the shell has constant thickness

$$
2 \varepsilon>0,
$$

where the parameter $\varepsilon$ is small enough compared with the radii of curvature of $S$, so that the mapping

$$
\Theta \in \mathcal{C}^{1}\left(\overline{\Omega^{\varepsilon}} ; \mathbb{E}^{3}\right),
$$

defined by

$$
\left.\Omega^{\varepsilon}:=\omega \times\right]-\varepsilon, \varepsilon[
$$

and

$$
\boldsymbol{\Theta}(x):=\boldsymbol{\theta}(y)+x_{3} \boldsymbol{a}_{3}(y) \text { for all } x=\left(y, x_{3}\right) \in \bar{\omega} \times[-\varepsilon, \varepsilon],
$$

is an immersion at all points of $\overline{\Omega^{\varepsilon}}$. That such small $\varepsilon>0$ exists has been proven in Ciarlet [5, Theorem 4.1-1]. Then the (stress-free) reference configuration of the shell with middle surface $S$ and thickness $2 \varepsilon$ is the closure of the open set

$$
\Theta\left(\Omega^{\varepsilon}\right) \subset \mathbb{E}^{3} .
$$

We assume that the shell satisfies a boundary condition of place on a subset $\Theta\left(\Gamma_{0}^{\varepsilon}\right)$ of its boundary, where

$$
\left.\Gamma_{0}^{\varepsilon}:=\gamma_{0} \times\right]-\varepsilon, \varepsilon[,
$$


and $\gamma_{0} \subset \partial \omega$ is a non-empty relatively open subset of the boundary of $\omega$, and that it is subjected to body forces whose densities per unit volume in $\Theta\left(\Omega^{\varepsilon}\right)$ are the vector fields

$$
\boldsymbol{f} \in L^{1}\left(\Omega^{\varepsilon} ; \mathbb{E}^{3}\right) .
$$

Note that surface forces can also be added on the lateral face $\boldsymbol{\Theta}(\partial \omega \times]-\varepsilon, \varepsilon[)$ of the shell with minor modifications of the ensuing analysis, but we do not consider them here for simplicity.

We assume that the deformations of the shell, henceforth denoted

$$
\tilde{\Theta}: \overline{\Omega^{\varepsilon}} \rightarrow \mathbb{E}^{3},
$$

satisfy the boundary condition of place

$$
\tilde{\Theta}=\Theta \text { on } \Gamma_{0}^{\varepsilon},
$$

and that they are of Kirchhoff-Love type; this means that there exist two vector fields $\tilde{\boldsymbol{\theta}}: \omega \rightarrow \mathbb{E}^{3}$ and $\tilde{\boldsymbol{\eta}}: \omega \rightarrow \mathbb{E}^{3}$ of class $W^{1,4}\left(\omega ; \mathbb{E}^{3}\right) \subset \mathcal{C}^{0}\left(\bar{\omega} ; \mathbb{E}^{3}\right)$, satisfying the conditions

$$
|\tilde{\boldsymbol{\eta}}|=1 \text { in } \omega \text { and } \tilde{\boldsymbol{\eta}} \cdot \partial_{\alpha} \tilde{\boldsymbol{\theta}}=0 \text { a.e. in } \omega
$$

such that

$$
\tilde{\boldsymbol{\Theta}}(x):=\tilde{\boldsymbol{\theta}}(y)+x_{3} \tilde{\boldsymbol{\eta}}(y) \text { for all } x=\left(y, x_{3}\right) \in \Omega^{\varepsilon} .
$$

In Sect. 2, we assume that the vector fields $\tilde{\boldsymbol{\theta}}$ and $\tilde{\boldsymbol{\eta}}$ satisfy in addition (see Definition 1)

$$
\left(\partial_{1} \tilde{\boldsymbol{\theta}} \wedge \partial_{2} \tilde{\boldsymbol{\theta}}\right) \cdot \tilde{\boldsymbol{\eta}}>0 \text { a.e. in } \omega
$$

or equivalently, that

$$
\tilde{\boldsymbol{\eta}}=\tilde{\boldsymbol{a}}_{3}:=\frac{\tilde{\boldsymbol{a}}_{1} \wedge \tilde{\boldsymbol{a}}_{2}}{\left|\tilde{\boldsymbol{a}}_{1} \wedge \tilde{\boldsymbol{a}}_{2}\right|} \text { a.e. in } \omega \text {. }
$$

In Sect. 3, we assume that the vector fields $\tilde{\boldsymbol{\theta}}$ and $\tilde{\boldsymbol{\eta}}$ satisfy in addition (see Definition 3)

$$
\left(\partial_{1} \tilde{\boldsymbol{\Theta}} \wedge \partial_{2} \tilde{\boldsymbol{\Theta}}\right) \cdot \tilde{\boldsymbol{\eta}}>0 \text { a.e. in } \Omega^{\varepsilon},
$$

or equivalently, that

$$
\operatorname{det} \nabla \tilde{\Theta}>0 \text { a.e. in } \Omega^{\varepsilon} \text {. }
$$

Finally, we assume that the (second Piola-Kirchhoff) stress tensor field associated with the deformation $\tilde{\boldsymbol{\Theta}}$, whose contravariant components are henceforth denoted

$$
\left(\tilde{\Sigma}^{i j}\right)_{i, j=1}^{3}: \Omega^{\varepsilon} \rightarrow \mathbb{S}^{3},
$$

is of F. John type; this means that the stress tensor field inside the shell is planar and parallel to the middle surface, so that $\tilde{\Sigma}^{i 3}=\tilde{\Sigma}^{3 i}=0$ for all $i=1,2,3$ (cf. Refs. [11, 12]).

A shell satisfying all these assumptions can be modeled by the nonlinear shell model of W.T. Koiter (Sect. 2). But this model does not necessarily possess a 
solution; in fact, an existence theorem is yet to be proven and has been an open problem for a long time.

In this paper, we remediate this situation by introducing two new shell models (Definition 1 and Definition 3), both of which are proven to be close in a specific sense to the nonlinear shell model of W.T. Koiter (cf. Sect. 2 and Sect. 3), and one of which is proven to have a solution (cf. Sect. 4).

We conclude this introduction by specifying the notation. All scalars used in this paper are real. $\mathbb{E}^{3}$ denotes the three-dimensional Euclidean space. $\mathbb{M}^{k \times \ell}$ denotes the spaces of matrices with $k$ rows and $\ell$ columns,

$$
\begin{aligned}
\mathbb{M}^{n} & :=\mathbb{M}^{n \times n}, \\
\mathbb{M}_{+}^{n} & :=\left\{F \in \mathbb{M}^{n} ; \operatorname{det} F>0\right\}, \\
S^{n} & :=\left\{F \in \mathbb{M}^{n} ; F^{T}=F\right\}, \\
\mathbb{S}_{>}^{n} & :=\left\{F \in \mathbb{S}^{n} ; F \text { is positive-definite }\right\},
\end{aligned}
$$

where $F^{T}$ denotes the transpose of the matrix $F$. The notation $\left(F_{i j}\right)$ designates a matrix whose component at the $i$-th row and $j$-th column is $F_{i j}$. Likewise, the notation $\left(F_{j}^{i}\right)$ designates a matrix whose component at the $i$-th row and $j$-th column is $F_{j}^{i}$.

The Euclidean inner and vector products of two vectors $u=\left(u_{i}\right)_{i=1}^{3}$ and $v=\left(v_{i}\right)_{i=1}^{3}$ are denoted and defined by

$$
u \cdot v:=\sum_{i} u_{i} v_{i} \text { and } u \wedge v:=\left(u_{2} v_{3}-u_{3} v_{2}, u_{3} v_{1}-u_{1} v_{3}, u_{1} v_{2}-u_{2} v_{1}\right)^{T} .
$$

The inner product of two matrices $A=\left(A_{j}^{i}\right)$ and $B=\left(B_{j}^{i}\right)$ is denoted and defined by

$$
A: B:=\sum_{i, j} A_{j}^{i} B_{j}^{i} .
$$

The Euclidean norm of a vector $v$ and the Frobenius norm of a matrix $F$ are respectively denoted and defined by

$$
|v|:=\sqrt{v \cdot v} \text { and }\|F\|:=\sqrt{F: F} .
$$

Throughout this paper, vector-valued functions and matrix-valued functions are respectively referred to as vector fields and tensor fields; they are denoted by boldface letters, to distinguish them from (scalar) functions, vectors, and matrices.

A vector field $\boldsymbol{v}=\left(v_{i}\right): \omega \rightarrow \mathbb{E}^{3}$ belongs to the Sobolev space $W^{k, p}\left(\omega ; \mathbb{E}^{3}\right)$, where $k \in \mathbb{N}$ and $1 \leq p<\infty$, if $v_{i} \in W^{k, p}(\omega)$ for all $i$; then its norm is defined by

$$
\|\boldsymbol{v}\|_{W^{k, p}\left(\omega ; \mathbb{E}^{3}\right)}:=\left(\sum_{|\alpha| \leq k} \int_{\omega}\left|\partial^{(\alpha)} \boldsymbol{v}(y)\right|^{p} d y\right)^{1 / p},
$$

where $\alpha=\left(\alpha_{1}, \alpha_{2}\right) \in \mathbb{N} \times \mathbb{N}$ is a multi-index, $|\alpha|:=\alpha_{1}+\alpha_{2}$, and $\partial^{(\alpha)} \boldsymbol{v}:=$ $\left(\partial^{(\alpha)} v_{i}\right)_{i=1}^{3}$, where $\partial^{(\alpha)} v_{i}:=\frac{\partial^{|\alpha|} v_{i}}{\partial y_{1}^{\alpha_{1}} \partial y_{2}^{\alpha_{2}}}$ if $|\alpha|>0$, and $\partial^{(\alpha)} v_{i}=v_{i}$ if $|\alpha|=0$. 
Likewise, a matrix field $\boldsymbol{F}=\left(F_{i j}\right): \omega \rightarrow \mathbb{M}^{n}$ belongs to the Sobolev space $W^{k, p}\left(\omega ; \mathbb{M}^{n}\right)$ if $F_{i j} \in W^{k, p}(\omega)$ for all $i, j=1, \ldots, n$; then its norm is defined by

$$
\|\boldsymbol{F}\|_{W^{k, p}\left(\omega ; \mathbb{M}^{n}\right)}:=\left(\sum_{|\alpha| \leq k} \int_{\omega}\left\|\partial^{(\alpha)} \boldsymbol{F}(y)\right\|^{p} d y\right)^{1 / p},
$$

where $\partial^{(\alpha)} \boldsymbol{F}:=\left(\partial^{(\alpha)} F_{i j}\right)_{i, j=1}^{n}$.

The $L^{\infty}$-norm of a vector field $\boldsymbol{v}$ or matrix field $\boldsymbol{F}$ is defined as the $L^{\infty}$-norm of the corresponding scalar functions $|\boldsymbol{v}|$ and $\|\boldsymbol{F}\|$.

Strong and weak convergences in Lebesgue and Sobolev spaces are respectively denoted by

$$
\rightarrow \text { and } \rightarrow \text {. }
$$

Partial derivatives of the first order, in the classical or distributional sense, are denoted by (recall that $y=\left(y_{\alpha}\right)$ denotes a generic point in $\bar{\omega}$ and $x=\left(y, x_{3}\right)$ denotes a generic point in $\overline{\Omega^{\varepsilon}}$ )

$$
\partial_{\alpha}:=\frac{\partial}{\partial y_{\alpha}}, \alpha=1,2, \quad \text { and } \partial_{3}:=\frac{\partial}{\partial x_{3}} .
$$

If not otherwise specified in the text, Greek indices range in the set $\{1,2\}$, Latin indices range in the set $\{1,2,3\}$, and the repeated index summation is systematically used in conjunction with these rules.

\section{First nonlinear shell model of Kirchhoff-Love type}

The objective of this section is to define a nonlinear shell model (Definition 1), similar to the nonlinear model of three-dimensional elasticity of Saint Venant Kirchhoff, but that is simpler and is valid only for deformations of KirchhhoffLove type. To this end, firstly, we will replace the Saint Venant - Kirchhoff stored energy function $W_{\text {SVK }}$ by a new stored energy function $W^{\varepsilon}$ by using an argument inspired by one in [15, Sect. 6], and, secondly, we will restrict the minimization set to deformations of Kirchhoff-Love type. Then we will prove that this new nonlinear shell model coincide to within the first order to the nonlinear shell model of W.T. Koiter (Definition 2) by means of formal asymptotic expansions with explicit estimates for the higher order terms.

With the immersion $\Theta$ defining the reference configuration of the shell (see Sect. 1), we associate the symmetric positive definite matrix fields $\left(g_{i j}\right)$ and $\left(g^{i j}\right)$ in $\mathcal{C}^{0}\left(\overline{\Omega^{\varepsilon}} ; \mathbb{S}_{>}^{3}\right)$ and the function $g \in \mathcal{C}^{0}\left(\overline{\Omega^{\varepsilon}}\right)$ by letting

$$
g_{i j}:=\partial_{i} \Theta \cdot \partial_{j} \boldsymbol{\Theta}, \quad\left(g^{i j}\right):=\left(g_{i j}\right)^{-1} \text { and } g:=\operatorname{det}\left(g_{i j}\right) .
$$

Note that the functions $g_{i j}$, resp. $g^{i j}$, are the covariant, resp. the contravariant, components of the metric tensor field associated with the immersion $\Theta$, and 
that $\sqrt{g(x)} d x$ is the unit volume element in the reference configuration $\Theta\left(\Omega^{\varepsilon}\right)$ of the shell.

Consider a shell satisfying the assumptions of Section 1. Assume in addition that the constitutive law of the elastic material constituting the shell is linear, in the sense that the stress tensor field $\left(\Sigma^{i j}(\boldsymbol{\Phi})\right): \Omega^{\varepsilon} \rightarrow \mathbb{S}^{3}$ and the strain tensor field $\left(E_{i j}(\boldsymbol{\Phi})\right): \Omega^{\varepsilon} \rightarrow \mathbb{S}^{3}$ associated with an arbitrary (i.e., not necessarily of Kirchhoff-Love type) deformation $\Phi: \Omega^{\varepsilon} \rightarrow \mathbb{E}^{3}$ are related to one another by the Saint Venant - Kirchhoff formula:

$$
\Sigma^{i j}(\boldsymbol{\Phi}):=\left(\lambda g^{i j} g^{k \ell}+\mu\left(g^{i k} g^{j \ell}+g^{i \ell} g^{j k}\right)\right) E_{k \ell}(\boldsymbol{\Phi}),
$$

where

$$
E_{k \ell}(\boldsymbol{\Phi}):=\frac{1}{2}\left(\partial_{k} \boldsymbol{\Phi} \cdot \partial_{\ell} \boldsymbol{\Phi}-g_{k \ell}\right) .
$$

Then, according to the three-dimensional elasticity theory, the deformation $\boldsymbol{\Phi}^{*}$ of the shell arising in response to applied body forces with density $\boldsymbol{f}$ should be a minimizer of the functional

$$
J_{\mathrm{SVK}}(\boldsymbol{\Phi}):=\int_{\Omega^{\varepsilon}} W_{\mathrm{SVK}}(\boldsymbol{\Phi}) \sqrt{g} d x-\int_{\Omega^{\varepsilon}} \boldsymbol{f} \cdot \boldsymbol{\Phi} \sqrt{g} d x,
$$

where

$$
W_{\mathrm{SVK}}(\boldsymbol{\Phi}):=\frac{1}{2} \Sigma^{i j}(\boldsymbol{\Phi}) E_{i j}(\boldsymbol{\Phi}),
$$

over the set

$$
U_{\mathrm{SVK}}\left(\Omega^{\varepsilon}\right):=\left\{\boldsymbol{\Phi} \in W^{1,4}\left(\Omega^{\varepsilon} ; \mathbb{E}^{3}\right) ; \boldsymbol{\Phi}=\boldsymbol{\Theta} \text { on } \Gamma_{0}^{\varepsilon}\right\} .
$$

Using the definition of the immersion $\Theta$ defining the reference configuration of the shell (Sect. 1), which implies in particular that $g^{\alpha 3}=g^{3 \alpha}=0$ and $g^{33}=1$, one deduces from the above Saint Venant - Kirchhoff formula that

$$
E_{\alpha 3}=\frac{1}{2 \mu} g_{\alpha \beta} \Sigma^{\beta 3}(\mathbf{\Phi})
$$

and

$$
E_{33}(\boldsymbol{\Phi})=\frac{1}{\lambda+2 \mu}\left(\Sigma^{33}(\boldsymbol{\Phi})-\lambda g^{\sigma \tau} E_{\sigma \tau}(\boldsymbol{\Phi})\right)
$$

then that

$$
\begin{aligned}
J_{\mathrm{SVK}}(\boldsymbol{\Phi}) & :=\frac{1}{4} \int_{\Omega^{\varepsilon}} g^{\alpha \beta \sigma \tau} E_{\sigma \tau}(\mathbf{\Phi}) E_{\alpha \beta}(\boldsymbol{\Phi}) \sqrt{g} d x-\int_{\Omega^{\varepsilon}} \boldsymbol{f} \cdot \boldsymbol{\Phi} \sqrt{g} d x \\
& +\frac{1}{2 \mu} \int_{\Omega^{\varepsilon}} g_{\alpha \beta} \Sigma^{\alpha 3}(\boldsymbol{\Phi}) \Sigma^{\beta 3}(\boldsymbol{\Phi}) \sqrt{g} d x \\
& +\frac{1}{2(\lambda+2 \mu)} \int_{\Omega^{\varepsilon}}\left(\Sigma^{33}(\boldsymbol{\Phi})\right)^{2} \sqrt{g} d x
\end{aligned}
$$

where

$$
g^{\alpha \beta \sigma \tau}:=\frac{4 \lambda \mu}{\lambda+2 \mu} g^{\alpha \beta} g^{\sigma \tau}+2 \mu\left(g^{\alpha \sigma} g^{\beta \tau}+g^{\alpha \tau} g^{\beta \sigma}\right) \text { in } \overline{\Omega^{\varepsilon}}
$$


Together with the assumption (made in Sect. 1) that the stress tensor field inside of a shell should be of John's type, this particular expression of Saint Venant - Kirchhoff functional $J_{\mathrm{SVK}}$ motivates the introduction of the following constitutive law for Kirchhoff-Love deformations of a shell: The stress tensor field $\left(\tilde{\Sigma}^{i j}\right): \Omega^{\varepsilon} \rightarrow \mathbb{S}^{3}$ and the strain tensor field $\left(\tilde{E}_{i j}\right): \Omega^{\varepsilon} \rightarrow \mathbb{S}^{3}$ associated with a deformation $\tilde{\boldsymbol{\Theta}}: \Omega^{\varepsilon} \rightarrow \mathbb{E}^{3}$ of Kirchhoff-Love type, i.e., a deformation of the form (see Sect. 1)

$$
\begin{gathered}
\tilde{\boldsymbol{\Theta}}(x):=\tilde{\boldsymbol{\theta}}(y)+x_{3} \tilde{\boldsymbol{a}}_{3}(y) \text { for all } x=\left(y, x_{3}\right) \in \Omega^{\varepsilon}, \\
\text { where } \tilde{\boldsymbol{a}}_{3}:=\frac{\partial_{1} \tilde{\boldsymbol{\theta}} \wedge \partial_{2} \tilde{\boldsymbol{\theta}}}{\left|\partial_{1} \tilde{\boldsymbol{\theta}} \wedge \partial_{2} \tilde{\boldsymbol{\theta}}\right|} \text { a.e. in } \omega
\end{gathered}
$$

are given by the relations

$$
\begin{aligned}
\tilde{\Sigma}^{\alpha \beta} & :=\frac{1}{2} g^{\alpha \beta \sigma \tau} \tilde{E}_{\sigma \tau}, \\
\tilde{\Sigma}^{\alpha 3}=\tilde{\Sigma}^{3 \alpha}: & :=0, \\
\tilde{\Sigma}^{33} & :=0,
\end{aligned}
$$

and

$$
\begin{aligned}
& \tilde{E}_{\alpha \beta}:=\frac{1}{2}\left(\partial_{\alpha} \tilde{\boldsymbol{\Theta}} \cdot \partial_{\beta} \tilde{\boldsymbol{\Theta}}-g_{\alpha \beta}\right), \\
& \tilde{E}_{\alpha 3}=\tilde{E}_{3 \alpha}:=0, \\
& \tilde{E}_{33}:=-\frac{\lambda g^{\sigma \tau}}{2(\lambda+2 \mu)}\left(\partial_{\sigma} \tilde{\boldsymbol{\Theta}} \cdot \partial_{\tau} \tilde{\boldsymbol{\Theta}}-g_{\sigma \tau}\right) .
\end{aligned}
$$

Remark 1 The strain energy density corresponding to the above stress and strain tensors is defined by

$$
W^{\varepsilon}(\tilde{\boldsymbol{\Theta}}):=\frac{1}{2} \tilde{\Sigma}^{\alpha \beta} \tilde{E}_{\alpha \beta}=\frac{1}{4} g^{\alpha \beta \sigma \tau} \tilde{E}_{\sigma \tau} \tilde{E}_{\alpha \beta},
$$

where the fourth-order tensor field $g^{\alpha \beta \sigma \tau}$ is defined in Definition 1 below.

Remark 2 The above expressions of $\tilde{\Sigma}^{i j}, \tilde{E}_{i j}$ and $W^{\varepsilon}$ are particular cases of the corresponding expressions given in [15, Theorem 6.1] for more general deformations of type

$$
\tilde{\boldsymbol{\Theta}}(x)=\tilde{\boldsymbol{\theta}}(y)+x_{3} \tilde{\boldsymbol{\eta}}(y) \text { a.e. } x=\left(y, x_{3}\right) \in \Omega^{\varepsilon},
$$

obtained by letting $\tilde{\boldsymbol{\eta}}=\tilde{\boldsymbol{a}}_{3}$ (as is the case for Kirchhoff-Love deformations).

Thanks to the above definitions, we are now in a position to define a minimization problem that constitutes a new nonlinear shell model. Then we will show how this new model is related to the well-known nonlinear shell model of W.T. Koiter. Recall that $\tilde{\boldsymbol{a}}_{3}$ is defined in terms of $\tilde{\boldsymbol{\theta}}$ by $\tilde{\boldsymbol{a}}_{3}:=\frac{\partial_{1} \tilde{\boldsymbol{\theta}} \wedge \partial_{2} \tilde{\boldsymbol{\theta}}}{\left|\partial_{1} \tilde{\boldsymbol{\theta}} \wedge \partial_{2} \tilde{\boldsymbol{\theta}}\right|}$ (see Section 1), and that it coincides with the (unique) unit normal vector field $\tilde{\boldsymbol{\eta}}: \omega \rightarrow \mathbb{E}^{3}$ to the surface $\tilde{\boldsymbol{\theta}}(\omega)$ that satisfies

$$
\left(\partial_{1} \tilde{\boldsymbol{\theta}} \wedge \partial_{2} \tilde{\boldsymbol{\theta}}\right) \cdot \tilde{\boldsymbol{\eta}}>0 \text { a.e. in } \omega \text {. }
$$


Definition 1 (first nonlinear shell model of Kirchhoff-Love type) Consider a shell with middle surface $\boldsymbol{\theta}(\bar{\omega}) \subset \mathbb{E}^{3}$ and thickness $2 \varepsilon>0$ that satisfies the assumptions of Sect. 1. The Lamé constants of the elastic material constituting the shell being denoted by $\lambda>0$ and $\mu>0$, let

$$
g^{\alpha \beta \sigma \tau}:=\frac{4 \lambda \mu}{\lambda+2 \mu} g^{\sigma \tau} g^{\alpha \beta}+2 \mu\left(g^{\alpha \sigma} g^{\beta \tau}+g^{\alpha \tau} g^{\beta \sigma}\right) \text { in } \overline{\Omega^{\varepsilon}} .
$$

Define the set

$$
\begin{aligned}
U(\omega):=\left\{\tilde{\boldsymbol{\theta}} \in W^{1,4}\left(\omega ; \mathbb{E}^{3}\right) ; \partial_{1} \tilde{\boldsymbol{\theta}} \wedge \partial_{2} \tilde{\boldsymbol{\theta}} \neq \mathbf{0} \text { a.e. in } \omega,\right. \\
\left.\quad \tilde{\boldsymbol{a}}_{3} \in W^{1,4}\left(\omega ; \mathbb{E}^{3}\right), \tilde{\boldsymbol{\theta}}=\boldsymbol{\theta} \text { and } \tilde{\boldsymbol{a}}_{3}=\boldsymbol{a}_{3} \text { on } \gamma_{0}\right\},
\end{aligned}
$$

then the set

$$
\begin{array}{r}
U\left(\Omega^{\varepsilon}\right):=\left\{\tilde{\boldsymbol{\Theta}} \in W^{1,4}\left(\Omega^{\varepsilon} ; \mathbb{E}^{3}\right) ; \exists \tilde{\boldsymbol{\theta}} \in U(\omega)\right. \text { such that } \\
\left.\tilde{\boldsymbol{\Theta}}\left(y, x_{3}\right)=\tilde{\boldsymbol{\theta}}(y)+x_{3} \tilde{\boldsymbol{a}}_{3}(y) \text { for all }\left(y, x_{3}\right) \in \Omega^{\varepsilon}\right\} .
\end{array}
$$

Given any $\tilde{\boldsymbol{\Theta}} \in U\left(\Omega^{\varepsilon}\right)$, let

$$
\tilde{E}_{\alpha \beta}:=\frac{1}{2}\left(\partial_{\alpha} \tilde{\boldsymbol{\Theta}} \cdot \partial_{\beta} \tilde{\boldsymbol{\Theta}}-g_{\alpha \beta}\right) \in L^{2}\left(\Omega^{\varepsilon}\right),
$$

let

$$
W^{\varepsilon}(\tilde{\boldsymbol{\Theta}}):=\frac{1}{4} g^{\alpha \beta \sigma \tau} \tilde{E}_{\sigma \tau} \tilde{E}_{\alpha \beta} \in L^{1}\left(\Omega^{\varepsilon}\right),
$$

and let

$$
J^{\varepsilon}(\tilde{\boldsymbol{\Theta}}):=\int_{\Omega^{\varepsilon}} W^{\varepsilon}(\tilde{\mathbf{\Theta}}) \sqrt{g} d x-\int_{\Omega^{\varepsilon}} \boldsymbol{f} \cdot \tilde{\boldsymbol{\Theta}} \sqrt{g} d x \in \mathbb{R} .
$$

Then the unknown deformation $\Theta^{*}: \Omega^{\varepsilon} \rightarrow \mathbb{E}^{3}$ of the shell can be found by solving the minimization problem:

$$
\text { Find } \boldsymbol{\Theta}^{*} \in U\left(\Omega^{\varepsilon}\right) \text { such that } J^{\varepsilon}\left(\boldsymbol{\Theta}^{*}\right) \leq J^{\varepsilon}(\tilde{\boldsymbol{\Theta}}) \text { for all } \tilde{\boldsymbol{\Theta}} \in U\left(\Omega^{\varepsilon}\right) \text {. }
$$

In order to define the nonlinear shell model of W.T.Koiter, we briefly recall a few definitions from differential geometry of surfaces. With the immersion $\boldsymbol{\theta}: \bar{\omega} \rightarrow \mathbb{E}^{3}$ defining the middle surface of the reference configuration of the shell (Sect. 1), we associate the functions

$$
\begin{aligned}
& a_{\alpha \beta}:=\boldsymbol{a}_{\alpha} \cdot \boldsymbol{a}_{\beta}, \\
& b_{\alpha \beta}:=-\boldsymbol{a}_{\alpha} \cdot \partial_{\beta} \boldsymbol{a}_{3}=-\partial_{\alpha} \boldsymbol{a}_{3} \cdot \boldsymbol{a}_{\beta}, \\
& c_{\alpha \beta}:=\partial_{\alpha} \boldsymbol{a}_{3} \cdot \partial_{\beta} \boldsymbol{a}_{3},
\end{aligned}
$$

which are precisely the covariant components of respectively the first, second, and third, fundamental forms of the surface $\boldsymbol{\theta}(\bar{\omega})$. Note that $\left(a_{\alpha \beta}\right) \in \mathcal{C}^{0}\left(\bar{\omega} ; \mathbb{S}_{>}^{2}\right)$, $\left(b_{\alpha \beta}\right) \in \mathcal{C}^{0}\left(\bar{\omega} ; \mathbb{S}^{2}\right)$ and $\left(c_{\alpha \beta}\right) \in \mathcal{C}^{0}\left(\bar{\omega} ; \mathbb{S}^{2}\right)$. 
Then we define the contravariant components $a^{\alpha \beta}$ of the above first fundamental form and the mixed components $b_{\beta}^{\alpha}$ of the above second fundamental form by

$$
\left(a^{\alpha \beta}\right):=\left(a_{\alpha \beta}\right)^{-1} \text { and } b_{\beta}^{\alpha}:=a^{\alpha \sigma} b_{\sigma \beta} \text {. }
$$

Finally, we let

$$
a:=\operatorname{det}\left(a_{\alpha \beta}\right),
$$

so that $\sqrt{a} d y$ is the unit area element along the surface $\boldsymbol{\theta}(\bar{\omega})$. Note that

$$
\left|\boldsymbol{a}_{1} \wedge \boldsymbol{a}_{2}\right|=\sqrt{a} \text { and } c_{\alpha \beta}=b_{\alpha \sigma} a^{\sigma \tau} b_{\tau \beta} \text { in } \bar{\omega} .
$$

With a mapping $\tilde{\boldsymbol{\theta}} \in W^{1,4}\left(\omega ; \mathbb{E}^{3}\right)$ that satisfies

$$
\tilde{\boldsymbol{a}}_{1} \wedge \tilde{\boldsymbol{a}}_{2} \neq \mathbf{0} \text { a.e. in } \omega \text { and } \tilde{\boldsymbol{a}}_{3} \in W^{1,4}\left(\omega ; \mathbb{E}^{3}\right),
$$

we likewise associate the functions

$$
\begin{aligned}
\tilde{a}_{\alpha \beta} & :=\tilde{\boldsymbol{a}}_{\alpha} \cdot \tilde{\boldsymbol{a}}_{\beta}, \\
\tilde{b}_{\alpha \beta} & :=-\tilde{\boldsymbol{a}}_{\alpha} \cdot \partial_{\beta} \tilde{\boldsymbol{a}}_{3}=-\partial_{\alpha} \tilde{\boldsymbol{a}}_{3} \cdot \tilde{\boldsymbol{a}}_{\beta}, \\
\tilde{c}_{\alpha \beta} & :=\partial_{\alpha} \tilde{\boldsymbol{a}}_{3} \cdot \partial_{\beta} \tilde{\boldsymbol{a}}_{3} .
\end{aligned}
$$

Note that $\left(\tilde{a}_{\alpha \beta}\right) \in L^{2}\left(\omega ; \mathbb{S}_{>}^{2}\right),\left(\tilde{b}_{\alpha \beta}\right) \in L^{2}\left(\omega ; \mathbb{S}^{2}\right)$ and $\left(\tilde{c}_{\alpha \beta}\right) \in L^{2}\left(\omega ; \mathbb{S}^{2}\right)$.

Then the nonlinear shell model proposed by W.T. Koiter in 1966 (Ref. [13]) reads as follows:

Definition 2 (nonlinear shell model of W.T. Koiter) Consider a shell with middle surface $\boldsymbol{\theta}(\bar{\omega}) \subset \mathbb{E}^{3}$ and thickness $2 \varepsilon>0$ that satisfies the assumptions of Sect. 1. The Lamé constants of the elastic material constituting the shell being denoted by $\lambda>0$ and $\mu>0$, and the density of the applied body being denoted by $\boldsymbol{f} \in L^{1}\left(\Omega^{\varepsilon} ; \mathbb{E}^{3}\right)$, let

$$
a^{\alpha \beta \sigma \tau}:=\frac{4 \lambda \mu}{\lambda+2 \mu} a^{\alpha \beta} a^{\sigma \tau}+2 \mu\left(a^{\alpha \sigma} a^{\beta \tau}+a^{\alpha \tau} a^{\beta \sigma}\right) \in \mathcal{C}^{0}(\bar{\omega}),
$$

and let

$$
\begin{aligned}
\boldsymbol{p}^{\varepsilon} & :=\int_{-\varepsilon}^{\varepsilon} \boldsymbol{f}\left(\cdot, x_{3}\right) \sqrt{g\left(\cdot, x_{3}\right)} d x_{3} \in L^{1}\left(\omega ; \mathbb{E}^{3}\right), \\
\boldsymbol{q}^{\varepsilon} & :=\int_{-\varepsilon}^{\varepsilon} x_{3} \boldsymbol{f}\left(\cdot, x_{3}\right) \sqrt{g\left(\cdot, x_{3}\right)} d x_{3} \in L^{1}\left(\omega ; \mathbb{E}^{3}\right) .
\end{aligned}
$$

Define the set (also used in Definition 1)

$$
\begin{aligned}
U(\omega):=\left\{\tilde{\boldsymbol{\theta}} \in W^{1,4}\left(\omega ; \mathbb{E}^{3}\right) ; \partial_{1} \tilde{\boldsymbol{\theta}} \wedge \partial_{2} \tilde{\boldsymbol{\theta}} \neq \mathbf{0} \text { a.e. in } \omega\right. \\
\left.\quad \tilde{\boldsymbol{a}}_{3} \in W^{1,4}\left(\omega ; \mathbb{E}^{3}\right), \tilde{\boldsymbol{\theta}}=\boldsymbol{\theta} \text { and } \tilde{\boldsymbol{a}}_{3}=\boldsymbol{a}_{3} \text { on } \gamma_{0}\right\} .
\end{aligned}
$$

Given any $\tilde{\boldsymbol{\theta}} \in U(\omega)$, let

$$
\begin{aligned}
W_{K}^{\varepsilon}(\tilde{\boldsymbol{\theta}}): & =\frac{\varepsilon}{8} a^{\alpha \beta \sigma \tau}\left(\tilde{a}_{\sigma \tau}-a_{\sigma \tau}\right)\left(\tilde{a}_{\alpha \beta}-a_{\alpha \beta}\right) \\
& +\frac{\varepsilon^{3}}{6} a^{\alpha \beta \sigma \tau}\left(\tilde{b}_{\sigma \tau}-b_{\sigma \tau}\right)\left(\tilde{b}_{\alpha \beta}-b_{\alpha \beta}\right),
\end{aligned}
$$


and let

$$
J_{K}^{\varepsilon}(\tilde{\boldsymbol{\theta}}):=\int_{\omega} W_{K}^{\varepsilon}(\tilde{\boldsymbol{\theta}}) \sqrt{a} d y-\int_{\omega}\left(\boldsymbol{p}^{\varepsilon} \cdot \tilde{\boldsymbol{\theta}}+\boldsymbol{q}^{\varepsilon} \cdot \tilde{\boldsymbol{a}}_{3}\right) d y .
$$

Then the unknown deformation $\boldsymbol{\theta}^{*}: \omega \rightarrow \mathbb{E}^{3}$ of the shell can by found by solving the minimization problem:

Find $\boldsymbol{\theta}^{*} \in U(\omega)$ such that $J_{K}^{\varepsilon}\left(\boldsymbol{\theta}^{*}\right) \leq J_{K}^{\varepsilon}(\tilde{\boldsymbol{\theta}})$ for all $\tilde{\boldsymbol{\theta}} \in U(\omega)$.

We now compare the nonlinear shell models of Definitions 1 and 2 . To this end, we use formal asymptotic expansions with respect to the transverse variable $x_{3} \in[-\varepsilon, \varepsilon]$ to identify the leading terms of the functional $J^{\varepsilon}$ appearing in Definition 1. This is legit since the shell is assumed to be thin, so $\varepsilon$, hence $x_{3}$, is as small as we wish.

Using the definition of the immersion $\Theta: \overline{\Omega^{\varepsilon}} \rightarrow \mathbb{E}^{3}$ in terms of the immersion $\boldsymbol{\theta}: \bar{\omega} \rightarrow \mathbb{E}^{3}$ (see Sect. 1), we deduce that, for all $\left.x_{3} \in\right]-\varepsilon, \varepsilon[$,

$$
g_{\alpha \beta}\left(\cdot, x_{3}\right)=a_{\alpha \beta}-2 x_{3} b_{\alpha \beta}+x_{3}^{2} c_{\alpha \beta} \text { in } \bar{\omega},
$$

which in turn implies that

$$
g^{\alpha \beta}\left(\cdot, x_{3}\right)=a^{\alpha \beta}+2 x_{3} a^{\alpha \sigma} b_{\sigma \tau} a^{\tau \beta}+x_{3}^{2} a^{\alpha \sigma} c_{\sigma \tau} a^{\tau \beta}+\ldots \text { in } \bar{\omega},
$$

where the dots replace the usual remainder in the Taylor power expansion of $g^{\alpha \beta}\left(\cdot, x_{3}\right)$.

Using these relations in the expression of the fourth-order tensor $g^{\alpha \beta \sigma \tau}$ (see Definition 1), we deduce that, for all $\left.x_{3} \in\right]-\varepsilon, \varepsilon[$ and a.e. in $\omega$,

$$
\begin{aligned}
\left(W^{\varepsilon}(\tilde{\boldsymbol{\Theta}}) \sqrt{g}\right)\left(\cdot, x_{3}\right) & =\frac{1}{4}\left(g^{\alpha \beta \sigma \tau}\left(\cdot, x_{3}\right) \sqrt{g\left(\cdot, x_{3}\right)}\right) \tilde{E}_{\sigma \tau}\left(\cdot, x_{3}\right) \tilde{E}_{\alpha \beta}\left(\cdot, x_{3}\right) \\
& =\frac{1}{4}\left(a^{\alpha \beta \sigma \tau} \sqrt{a}+r^{\alpha \beta \sigma \tau}\left(\cdot, x_{3}\right)\right) \tilde{E}_{\sigma \tau}\left(\cdot, x_{3}\right) \tilde{E}_{\alpha \beta}\left(\cdot, x_{3}\right),
\end{aligned}
$$

where $a^{\alpha \beta \sigma \tau}$ is the fourth order tensor appearing in Definition 2 and

$$
r^{\alpha \beta \sigma \tau}\left(\cdot, x_{3}\right):=\left(g^{\alpha \beta \sigma \tau} \sqrt{g}\right)\left(\cdot, x_{3}\right)-a^{\alpha \beta \sigma \tau} \sqrt{a} \text { in } \bar{\omega} .
$$

Note that, for all $y \in \bar{\omega},\left(a^{\alpha \beta \sigma \tau}(y) \sqrt{a(y)}\right)$ is the first term of the Taylor series of the function

$$
x_{3} \in[-\varepsilon, \varepsilon] \mapsto g^{\alpha \beta \sigma \tau}\left(y, x_{3}\right) \sqrt{g\left(y, x_{3}\right)} \in \mathbb{R},
$$

so that $r^{\alpha \beta \sigma \tau}\left(y, x_{3}\right)$ is nothing but the remainder term; therefore it is "negligible" in the above expression of $\left(W^{\varepsilon}(\tilde{\boldsymbol{\Theta}}) \sqrt{g}\right)$, in the sense that there exists a constant $C=C\left(\varepsilon_{0}, \boldsymbol{\theta}\right)$ such that, for all $0<\varepsilon \leq \varepsilon_{0}$ and for all $x_{3} \in[-\varepsilon, \varepsilon]$,

$$
\sup _{y \in \bar{\omega}}\left|r^{\alpha \beta \sigma \tau}\left(y, x_{3}\right)\right| \leq C x_{3} \leq C \varepsilon .
$$

Furthermore, since the Lamé constants $\lambda$ and $\mu$ are positive and since $\left(a_{\alpha \beta}\right) \in$ $\mathcal{C}^{0}\left(\bar{\omega} ; \mathbb{S}_{>}^{2}\right)$, there exists two constants $0<c_{0} \leq c_{1}<\infty$ such that

$$
c_{0}\left\|\left(t_{\alpha \beta}\right)\right\|^{2} \leq \frac{1}{4} a^{\alpha \beta \sigma \tau}(y) t_{\sigma \tau} t_{\alpha \beta} \sqrt{a(y)} \leq c_{1}\left\|\left(t_{\alpha \beta}\right)\right\|^{2}
$$

for all $y \in \bar{\omega}$ and for all matrices $\left(t_{\alpha \beta}\right) \in \mathbb{S}^{2}$. 
Remark 3 Inequalities (4) hold in particular for

$$
c_{0}=\mu \inf _{y \in \bar{\omega}} \frac{\lambda_{\min }(y)^{1 / 2}}{\lambda_{\max }(y)^{3 / 2}} \text { and } c_{1}=\mu \inf _{y \in \bar{\omega}} \frac{\lambda_{\max }(y)^{1 / 2}}{\lambda_{\min }(y)^{3 / 2}},
$$

where $\lambda_{\min }(y) \leq \lambda_{\max }(y)$ are the two eigenvalues of the positive definite symmetric matrix $\left(a_{\alpha \beta}(y)\right) \in \mathbb{S}_{>}^{2}$.

It follows that, on the one hand,

$$
\begin{aligned}
\left(W^{\varepsilon}(\tilde{\mathbf{\Theta}}) \sqrt{g}\right)\left(y, x_{3}\right) & =\frac{1}{4} a^{\alpha \beta \sigma \tau}(y) \tilde{E}_{\sigma \tau}\left(y, x_{3}\right) \tilde{E}_{\alpha \beta}\left(y, x_{3}\right) \sqrt{a(y)} \\
& +\frac{1}{4} r^{\alpha \beta \sigma \tau}\left(y, x_{3}\right) \tilde{E}_{\sigma \tau}\left(y, x_{3}\right) \tilde{E}_{\alpha \beta}\left(y, x_{3}\right),
\end{aligned}
$$

and, on the other hand, there exists a constant $\varepsilon_{0}>0$ such that, for all $\varepsilon \leq \varepsilon_{0}$, all $\left.x_{3} \in\right]-\varepsilon, \varepsilon[$, and a.e. $y \in \omega$,

$$
\begin{aligned}
& \left(W^{\varepsilon}(\tilde{\boldsymbol{\Theta}}) \sqrt{g}\right)\left(y, x_{3}\right) \geq \frac{c_{0}}{2}\left\|\left(\tilde{E}_{\alpha \beta}\left(y, x_{3}\right)\right)\right\|^{2}, \\
& \left(W^{\varepsilon}(\tilde{\boldsymbol{\Theta}}) \sqrt{g}\right)\left(y, x_{3}\right) \leq 2 c_{1}\left\|\left(\tilde{E}_{\alpha \beta}\left(y, x_{3}\right)\right)\right\|^{2} .
\end{aligned}
$$

Using these inequalities and estimate (3) of the coefficients $r^{\alpha \beta \sigma \tau}\left(y, x_{3}\right)$ to estimate the last term in the right-hand side of (5), we deduce that the functional $J^{\varepsilon}$ appearing in Definition 1 satisfies, for all $0<\varepsilon \leq \varepsilon_{0}$,

$$
\begin{aligned}
J^{\varepsilon}(\tilde{\boldsymbol{\Theta}}) & =\frac{1}{4} \int_{\Omega^{\varepsilon}} a^{\alpha \beta \sigma \tau}(y) \tilde{E}_{\sigma \tau}\left(y, x_{3}\right) \tilde{E}_{\alpha \beta}\left(y, x_{3}\right) \sqrt{a(y)} d x-\int_{\Omega^{\varepsilon}} \boldsymbol{f} \cdot \tilde{\boldsymbol{\Theta}} \sqrt{g} d x \\
& +\varepsilon R^{\varepsilon}(\tilde{\mathbf{\Theta}}),
\end{aligned}
$$

where the last term in the above right-hand side satisfies

$$
\left|R^{\varepsilon}(\tilde{\boldsymbol{\Theta}})\right| \leq C\left\|\left(\tilde{E}_{\alpha \beta}\right)\right\|_{L^{2}\left(\Omega^{\varepsilon} ; \mathbb{S}^{2}\right)}^{2},
$$

for some constant $C$ independent of $\varepsilon$ and $\tilde{\boldsymbol{\Theta}}$; this means that the term " $\varepsilon R^{\varepsilon}(\tilde{\boldsymbol{\Theta}})$ " apearing in the right hand side of the above expression of $J^{\varepsilon}(\tilde{\boldsymbol{\Theta}})$ is negligible with respect to the first term of the same right hand side.

Next we infer from the definition (see Sect. 1) of the immersions $\Theta: \overline{\Omega^{\varepsilon}} \rightarrow \mathbb{E}^{3}$ and $\tilde{\boldsymbol{\Theta}}: \Omega^{\varepsilon} \rightarrow \mathbb{E}^{3}$ in terms of the immersions $\boldsymbol{\theta}: \bar{\omega} \rightarrow \mathbb{E}^{3}$ and $\tilde{\boldsymbol{\theta}}: \omega \rightarrow \mathbb{E}^{3}$ that the strain tensor field $\tilde{E}_{\alpha \beta}$ defined by (1) satisfies, at each $\left.x_{3} \in\right]-\varepsilon, \varepsilon[$,

$$
\begin{aligned}
\tilde{E}_{\alpha \beta}\left(\cdot, x_{3}\right) & =\frac{1}{2}\left(\partial_{\alpha}\left(\tilde{\boldsymbol{\theta}}+x_{3} \tilde{\boldsymbol{a}}_{3}\right) \cdot \partial_{\beta}\left(\tilde{\boldsymbol{\theta}}+x_{3} \tilde{\boldsymbol{a}}_{3}\right)-g_{\alpha \beta}\right) \\
& =\frac{1}{2}\left(\tilde{a}_{\alpha \beta}-a_{\alpha \beta}\right)-x_{3}\left(\tilde{b}_{\alpha \beta}-b_{\alpha \beta}\right)+\frac{x_{3}^{2}}{2}\left(\tilde{c}_{\alpha \beta}-c_{\alpha \beta}\right) \text { a.e. in } \omega .
\end{aligned}
$$

Then a series of straightforward computations based on the Fubini integral formula, on the symmetry of the interval $]-\varepsilon, \varepsilon[$, and on the symmetry relations 
$a^{\alpha \beta \sigma \tau}=a^{\sigma \tau \alpha \beta}$, show that

$$
\begin{aligned}
W_{0}^{\varepsilon}(\tilde{\boldsymbol{\theta}}):= & \frac{1}{4} \int_{-\varepsilon}^{\varepsilon} a^{\alpha \beta \sigma \tau}(y) \tilde{E}_{\sigma \tau}\left(y, x_{3}\right) \tilde{E}_{\alpha \beta}\left(y, x_{3}\right) \sqrt{a(y)} d x_{3} \\
=W_{K}^{\varepsilon}(\tilde{\boldsymbol{\theta}}) & +\frac{\varepsilon^{3}}{12} a^{\alpha \beta \sigma \tau}\left(\tilde{a}_{\sigma \tau}-a_{\sigma \tau}\right)\left(\tilde{c}_{\alpha \beta}-c_{\alpha \beta}\right) \\
& +\frac{\varepsilon^{5}}{40} a^{\alpha \beta \sigma \tau}\left(\tilde{c}_{\sigma \tau}-c_{\sigma \tau}\right)\left(\tilde{c}_{\alpha \beta}-c_{\alpha \beta}\right),
\end{aligned}
$$

where $W_{K}^{\varepsilon}(\tilde{\boldsymbol{\theta}})$ is the function defined in Definition 2 .

Using this relation in the previous expression of $J^{\varepsilon}(\tilde{\boldsymbol{\Theta}})$, we finally deduce that

$$
\begin{aligned}
J^{\varepsilon}(\tilde{\boldsymbol{\Theta}})=\int_{\omega} W_{0}^{\varepsilon}(\tilde{\boldsymbol{\theta}}) \sqrt{a} d y- & \int_{\omega}\left(\boldsymbol{p}^{\varepsilon} \cdot \tilde{\boldsymbol{\theta}}+\boldsymbol{q}^{\varepsilon} \cdot \tilde{\boldsymbol{a}}_{3}\right) d y+\varepsilon R^{\varepsilon}(\tilde{\boldsymbol{\Theta}}) \\
=J_{K}^{\varepsilon}(\tilde{\boldsymbol{\theta}})+\varepsilon R^{\varepsilon}(\tilde{\boldsymbol{\Theta}}) & +\frac{\varepsilon^{3}}{12} \int_{\omega} a^{\alpha \beta \sigma \tau}\left(\tilde{a}_{\sigma \tau}-a_{\sigma \tau}\right)\left(\tilde{c}_{\alpha \beta}-c_{\alpha \beta}\right) \sqrt{a} d y \\
& +\frac{\varepsilon^{5}}{40} \int_{\omega} a^{\alpha \beta \sigma \tau}\left(\tilde{c}_{\sigma \tau}-c_{\sigma \tau}\right)\left(\tilde{c}_{\alpha \beta}-c_{\alpha \beta}\right) \sqrt{a} d y .
\end{aligned}
$$

The last equality shows that the functional $J_{K}^{\varepsilon}$ of Definition 2 is obtained from the functional $J^{\varepsilon}$ of Definition 1 by dropping the last three terms of the right-hand side of (9). The reason from dropping $\varepsilon R^{\varepsilon}(\tilde{\boldsymbol{\Theta}})$ has been explained before: see (6) above. The reason for dropping the last two terms of (9) is that the third fundamental form of a surface is completely determined by the first and second fundamental forms of the same surface by means of the relation $c_{\alpha \beta}=b_{\alpha \sigma} a^{\sigma \tau} b_{\tau \beta}$ in the case of the surface $\boldsymbol{\theta}(\bar{\omega})$, and $\tilde{c}_{\alpha \beta}=\tilde{b}_{\alpha \sigma} \tilde{a}^{\sigma \tau} \tilde{b}_{\tau \beta}$ in the case of the surface $\tilde{\boldsymbol{\theta}}(\omega)$. This implies that (this formula has been used previously, in the proof of Theorem 4.3 in [7])

$$
\begin{aligned}
\left(\tilde{c}_{\alpha \beta}-c_{\alpha \beta}\right) & =\tilde{b}_{\alpha}^{\sigma}\left(\tilde{b}_{\sigma \beta}-b_{\sigma \beta}\right)+\left(\tilde{b}_{\alpha \tau}-b_{\alpha \tau}\right) b_{\beta}^{\tau}-\tilde{b}_{\alpha \tau} b_{\beta}^{\tau}+\tilde{b}_{\alpha}^{\sigma} b_{\sigma \beta} \\
& =\tilde{b}_{\alpha}^{\sigma}\left(\tilde{b}_{\sigma \beta}-b_{\sigma \beta}\right)+\left(\tilde{b}_{\alpha \tau}-b_{\alpha \tau}\right) b_{\beta}^{\tau}-\tilde{b}_{\alpha}^{\sigma}\left(\tilde{a}_{\sigma \tau}-a_{\sigma \tau}\right) b_{\beta}^{\tau},
\end{aligned}
$$

where $\tilde{b}_{\alpha}^{\tau}:=\tilde{a}^{\tau \sigma} \tilde{b}_{\sigma \alpha}$ and $\left(\tilde{a}^{\tau \sigma}\right):=\left(a_{\alpha \beta}\right)^{-1}$, which in turn implies that

$$
\left\|\left(\tilde{c}_{\alpha \beta}-c_{\alpha \beta}\right)\right\| \leq C\left(\left\|\left(\tilde{b}_{\beta}^{\alpha}\right)\right\|\right)\left(\left\|\left(\tilde{a}_{\alpha \beta}-a_{\alpha \beta}\right)\right\|+\left\|\left(\tilde{b}_{\alpha \beta}-b_{\alpha \beta}\right)\right\|\right)
$$

where the constant $C\left(\left\|\left(\tilde{b}_{\beta}^{\alpha}\right)\right\|\right)$ appearing in the right hand side is given by

$$
C\left(\left\|\left(\tilde{b}_{\beta}^{\alpha}\right)\right\|\right):=\left\|\left(b_{\beta}^{\alpha}\right)\right\|+\left(1+\left\|\left(b_{\beta}^{\alpha}\right)\right\|\right)\left\|\left(\tilde{b}_{\beta}^{\alpha}\right)\right\| .
$$

As a consequence, given any constant $M>1$, there exists a constant $C=$ $C\left(\varepsilon_{0}, \boldsymbol{\theta}, M\right)<\infty$ such that, on the one hand,

$\left|\frac{\varepsilon^{3}}{12} a^{\alpha \beta \sigma \tau}\left(\tilde{a}_{\sigma \tau}-a_{\sigma \tau}\right)\left(\tilde{c}_{\alpha \beta}-c_{\alpha \beta}\right)\right| \leq C \varepsilon\left(\varepsilon\left\|\left(\tilde{a}_{\alpha \beta}-a_{\alpha \beta}\right)\right\|^{2}+\varepsilon^{3}\left\|\left(\tilde{b}_{\alpha \beta}-b_{\alpha \beta}\right)\right\|^{2}\right)$, 
and

$\left|\frac{\varepsilon^{5}}{40} a^{\alpha \beta \sigma \tau}\left(\tilde{c}_{\sigma \tau}-c_{\sigma \tau}\right)\left(\tilde{c}_{\alpha \beta}-c_{\alpha \beta}\right)\right| \leq C \varepsilon^{2}\left(\varepsilon\left\|\left(\tilde{a}_{\alpha \beta}-a_{\alpha \beta}\right)\right\|^{2}+\varepsilon^{3}\left\|\left(\tilde{b}_{\alpha \beta}-b_{\alpha \beta}\right)\right\|^{2}\right)$,

for all $\varepsilon \leq \varepsilon_{0}$ and for all immersions $\tilde{\boldsymbol{\theta}}$ that satisfy

$$
\left\|\left(\tilde{b}_{\beta}^{\alpha}\right)\right\|_{L^{\infty}\left(\omega ; \mathbb{M}^{2}\right)} \leq M
$$

Since, on the other hand,

$$
W_{K}^{\varepsilon}(\tilde{\boldsymbol{\theta}}) \geq \frac{c_{0}}{2 \sup _{y \in \bar{\omega}} \sqrt{a(y)}}\left(\varepsilon\left\|\left(\tilde{a}_{\alpha \beta}-a_{\alpha \beta}\right)\right\|^{2}+\varepsilon^{3}\left\|\left(\tilde{b}_{\alpha \beta}-b_{\alpha \beta}\right)\right\|^{2}\right)
$$

(thanks to the coerciveness inequality (4) satisfied by the tensor field $\frac{1}{4} a^{\alpha \beta \sigma \tau} \sqrt{a}$ ), it follows that the last two terms of the right-hand side of (9) are "negligible" with respect to the integral $\int_{\omega} W_{K}^{\varepsilon}(\tilde{\boldsymbol{\theta}}) \sqrt{a} d y$ that appears in the definition of the functional $J_{K}^{\varepsilon}(\tilde{\boldsymbol{\theta}})$.

Thus, we showed by means of formal asymptotic expansions that the nonlinear shell models in Definitions 1 and 2 coincide to within the first order.

Note that both models are minimization problems that share the same set of admissible deformations, since

$$
\tilde{\boldsymbol{\Theta}} \in U\left(\Omega^{\varepsilon}\right) \Leftrightarrow \tilde{\boldsymbol{\theta}} \in U(\omega),
$$

and the same linear form modeling the applied forces, since

$$
\int_{\Omega^{\varepsilon}} \boldsymbol{f} \cdot \tilde{\boldsymbol{\Theta}} \sqrt{g} d x=\int_{\omega}\left(\boldsymbol{p}^{\varepsilon} \cdot \tilde{\boldsymbol{\theta}}+\boldsymbol{q}^{\varepsilon} \cdot \tilde{\boldsymbol{a}}_{3}\right) d y .
$$

Thus the sole difference between these two models is the definition of their respective two-dimensional strain energy density, viz., $\int_{-\varepsilon}^{\varepsilon} W^{\varepsilon}(\tilde{\mathbf{\Theta}}) \sqrt{g} d x_{3}$ for the shell model proposed in Definition 1 , and $W_{K}^{\varepsilon}(\tilde{\boldsymbol{\theta}})$ for Koiter's model (Definition 2). More specifically, since in view of (5) and (7) we have

$$
\begin{aligned}
\int_{-\varepsilon}^{\varepsilon} W^{\varepsilon}(\tilde{\boldsymbol{\Theta}}) \sqrt{g} d x_{3} & =W_{K}^{\varepsilon}(\tilde{\boldsymbol{\theta}}) \sqrt{a}+\frac{\varepsilon^{3}}{12} a^{\alpha \beta \sigma \tau}\left(\tilde{a}_{\sigma \tau}-a_{\sigma \tau}\right)\left(\tilde{c}_{\alpha \beta}-c_{\alpha \beta}\right) \\
& +\frac{\varepsilon^{5}}{40} a^{\alpha \beta \sigma \tau}\left(\tilde{c}_{\sigma \tau}-c_{\sigma \tau}\right)\left(\tilde{c}_{\alpha \beta}-c_{\alpha \beta}\right)+\frac{1}{4} \int_{-\varepsilon}^{\varepsilon} r^{\alpha \beta \sigma \tau} \tilde{E}_{\sigma \tau} \tilde{E}_{\alpha \beta} d x_{3},
\end{aligned}
$$

where $r^{\alpha \beta \sigma \tau}$ is the function defined by (2), the difference between these strain energy densities is given explicitly by

$$
\begin{aligned}
\int_{-\varepsilon}^{\varepsilon} W^{\varepsilon}(\tilde{\boldsymbol{\Theta}}) \sqrt{g} d x_{3}-W_{K}^{\varepsilon}(\tilde{\boldsymbol{\theta}}) \sqrt{a} & =\frac{1}{4} \int_{-\varepsilon}^{\varepsilon} r^{\alpha \beta \sigma \tau} \tilde{E}_{\sigma \tau} \tilde{E}_{\alpha \beta} d x_{3} \\
& +\frac{\varepsilon^{3}}{12} a^{\alpha \beta \sigma \tau}\left(\tilde{a}_{\sigma \tau}-a_{\sigma \tau}\right)\left(\tilde{c}_{\alpha \beta}-c_{\alpha \beta}\right) \sqrt{a} \\
& +\frac{\varepsilon^{5}}{40} a^{\alpha \beta \sigma \tau}\left(\tilde{c}_{\sigma \tau}-c_{\sigma \tau}\right)\left(\tilde{c}_{\alpha \beta}-c_{\alpha \beta}\right) \sqrt{a}
\end{aligned}
$$


Note that the right hand side is "negligible" with respect to Koiter's stored energy function $W_{K}^{\varepsilon}(\tilde{\boldsymbol{\theta}}) \sqrt{a}$ since the estimate (3) of $r^{\alpha \beta \sigma \tau}$ and the coerciveness inequality (4) of $a^{\alpha \beta \sigma \tau}$ together imply that there exists a constant $C_{1}$ depending only on $\varepsilon_{0}$ and $\boldsymbol{\theta}$ such that

$$
\left|\int_{-\varepsilon}^{\varepsilon} r^{\alpha \beta \sigma \tau} \tilde{E}_{\sigma \tau} \tilde{E}_{\alpha \beta} d x_{3}\right| \leq C_{1} \varepsilon\left|\frac{1}{4} \int_{-\varepsilon}^{\varepsilon} a^{\alpha \beta \sigma \tau} \tilde{E}_{\sigma \tau} \tilde{E}_{\alpha \beta} \sqrt{a} d x_{3}\right|=C_{1} \varepsilon W_{0}^{\varepsilon}(\tilde{\boldsymbol{\theta}}),
$$

where $W_{0}^{\varepsilon}(\tilde{\boldsymbol{\theta}})$ is defined by $(8)$. Combined with the previous estimates of the last two terms of the right-hand side of (8), this shows that, for all $\varepsilon<\varepsilon_{0}$ and for all immersions $\tilde{\boldsymbol{\theta}} \in U(\omega)$ such that

$$
\left\|\left(\tilde{b}_{\beta}^{\alpha}\right)\right\|_{L^{\infty}\left(\omega ; \mathbb{M}^{2}\right)} \leq M
$$

the difference between the two-dimensional strain energy densities of the shell models proposed in Definition 1 and Definition 2 satisfies

$$
\left|\int_{-\varepsilon}^{\varepsilon} W^{\varepsilon}(\tilde{\boldsymbol{\Theta}}) \sqrt{g} d x_{3}-W_{K}^{\varepsilon}(\tilde{\boldsymbol{\theta}}) \sqrt{a}\right| \leq C_{2} \varepsilon W_{K}^{\varepsilon}(\tilde{\boldsymbol{\theta}}) \sqrt{a}
$$

where the constant $C_{2}$ depends on $\varepsilon_{0}, \boldsymbol{\theta}$ and $M$.

The two nonlinear shell models presented in this section (Definitions 1 and 2) are the simplest nonlinear shell models that can be used for any type of shell, irrespectively of the geometry of their middle surface and of the boundary conditions of place used in the definition of the minimization set $U(\omega)$ (simpler models exists for particular kind of shells: see. e.g., Le Dret and Raoult [14], Friesecke, James, Mora and Müller [10], Bunoiu, Ciarlet and Mardare [4], Ciarlet and Mardare $[8,9]$ ). For this reason, Koiter's nonlinear shell model and its linear counterpart are probably the most commonly used shell models in theoretical and computational mechanics. But for Koiter's nonlinear shell model no existence theorem have been established so far. In fact, an existence theorem for this model, or even for the shell model of Definition 1 for that matter, seems unlikely at the present state of the theory. The main difficulty is that both models use the vector field

$$
\tilde{\boldsymbol{a}}_{3}:=\frac{\partial_{1} \tilde{\boldsymbol{\theta}} \wedge \partial_{2} \tilde{\boldsymbol{\theta}}}{\left|\partial_{1} \tilde{\boldsymbol{\theta}} \wedge \partial_{2} \tilde{\boldsymbol{\theta}}\right|}
$$

which makes it unlikely for the minimization set to be closed for any reasonable (for a minimization problem) weak topology. This is the reason why we define in the next section another nonlinear shell model, similar to that of Definition 1, but for which we can prove an existence theorem; cf. Section 4.

\section{Second nonlinear shell model of Kirchhoff-Love type}

The objective of this section is to define a new nonlinear shell model (Definition 3) that is similar to the one in Definition 1, but with the additional property 
that it possesses at least a solution; cf. Theorem 1. To this end, we will replace, firstly, the stored energy function $W^{\varepsilon}$ in Definition 1 by a new stored energy function $W_{\sharp}^{\varepsilon}$ of the form

$$
W_{\sharp}^{\varepsilon}=W^{\varepsilon}+\text { h.o.t., }
$$

where "h.o.t." stands for higher order terms with respect to the strain tensor field $\tilde{E}_{\alpha \beta}$ than those of $W^{\varepsilon}$, and, secondly, we will replace the minimization set $U\left(\Omega^{\varepsilon}\right)$ in Definition 1 by a new minimization set $U_{\sharp}\left(\Omega^{\varepsilon}\right)$ whose elements $\tilde{\boldsymbol{\Theta}}$ are orientation-preserving, i.e.,

$$
\operatorname{det} \nabla \tilde{\Theta}>0 \text { a.e. in } \Omega^{\varepsilon} \text {. }
$$

Note that the assumption that the mapping $\tilde{\boldsymbol{\Theta}}: \bar{\Omega} \rightarrow \mathbb{E}^{3}$ is orientationpreserving is desirable from a physical point of view. In fact, it would be desirable to impose in addition that the mapping $\tilde{\boldsymbol{\Theta}}$ is injective, since this means that no interpenetration of matter occurs; but this would be detrimental to proving an existence theorem, so for this reason we drop this assumption.

The strain energy function $W_{\sharp}^{\varepsilon}(\tilde{\boldsymbol{\Theta}})$ that we are introducing in Definition 3 below depends on the unknown deformation $\tilde{\Theta} \in U_{\sharp}\left(\Omega^{\varepsilon}\right)$ of the shell by means of the functions

$$
\tilde{g}_{\alpha \beta}:=\partial_{\alpha} \tilde{\boldsymbol{\Theta}} \cdot \partial_{\beta} \tilde{\boldsymbol{\Theta}} \text { a.e. in } \Omega^{\varepsilon} \text {. }
$$

Note that these functions are precisely the covariant components of the projection on the tangent planes to the surface $\tilde{\boldsymbol{\theta}}(\omega)$ of metric tensor field associated with $\tilde{\Theta}: \Omega^{\varepsilon} \rightarrow \mathbb{E}^{3}$. The other covariant components of the same metric tensor field are given by

$$
\partial_{\alpha} \tilde{\boldsymbol{\Theta}} \cdot \partial_{3} \tilde{\boldsymbol{\Theta}}=\partial_{3} \tilde{\boldsymbol{\Theta}} \cdot \partial_{\alpha} \tilde{\boldsymbol{\Theta}}=0 \text { and } \partial_{3} \tilde{\boldsymbol{\Theta}} \cdot \partial_{3} \tilde{\boldsymbol{\Theta}}=1 \text { a.e. in } \Omega^{\varepsilon} \text {, }
$$

as a consequence to the definition of the mapping $\tilde{\boldsymbol{\Theta}}$ as a mapping of the form (cf. Sect 1)

$$
\tilde{\boldsymbol{\Theta}}\left(y, x_{3}\right)=\tilde{\boldsymbol{\theta}}(y)+x_{3} \tilde{\boldsymbol{\eta}}(y) \text { for all }\left(y, x_{3}\right) \in \Omega^{\varepsilon},
$$

where the vector field $\tilde{\boldsymbol{\theta}}$ and $\tilde{\boldsymbol{\eta}}$ belong to the space $W^{1,4}\left(\omega ; \mathbb{E}^{3}\right)$ and satisfy

$$
|\tilde{\boldsymbol{\eta}}|=1 \text { in } \omega \text { and } \tilde{\boldsymbol{\eta}} \cdot \partial_{\alpha} \tilde{\boldsymbol{\theta}}=0 \text { a.e. in } \omega \text {. }
$$

Note also that the definition of the matrix field $\left(\tilde{g}_{\alpha \beta}\right): \Omega^{\varepsilon} \rightarrow \mathbb{S}^{2}$ implies that

$$
\begin{aligned}
\operatorname{det}\left(\tilde{g}_{\alpha \beta}\right) & =\operatorname{det}\left(\partial_{\alpha} \tilde{\boldsymbol{\Theta}} \cdot \partial_{\beta} \tilde{\boldsymbol{\Theta}}\right)=\operatorname{det}\left(\partial_{i} \tilde{\boldsymbol{\Theta}} \cdot \partial_{j} \tilde{\boldsymbol{\Theta}}\right) \\
& =\operatorname{det}\left((\nabla \tilde{\boldsymbol{\Theta}})^{T} \nabla \tilde{\boldsymbol{\Theta}}\right)=(\operatorname{det} \boldsymbol{\nabla} \tilde{\boldsymbol{\Theta}})^{2} \geq 0 \text { a.e. in } \Omega^{\varepsilon}
\end{aligned}
$$

which in turn implies that the matrix field $\left(g^{\alpha \sigma} \tilde{g}_{\sigma \beta}\right): \Omega^{\varepsilon} \rightarrow \mathbb{M}^{2}$, where $\left(g^{\alpha \sigma}(x)\right):=$ $\left(g_{\alpha \sigma}(x)\right)^{-1}$ for all $x \in \overline{\Omega^{\varepsilon}}$, satisfies

$$
\operatorname{det}\left(g^{\alpha \sigma} \tilde{g}_{\sigma \beta}\right) \geq 0 \text { a.e. in } \Omega^{\varepsilon} .
$$

We are now in a position to introduce our new nonlinear shell model. The choice of the strain energy function is inspired, but different, by a previous example in three-dimensional elasticity by Ciarlet and Geymonat [6] (see also Ciarlet and Mardare [9]). 
Definition 3 (nonlinear shell model of Kirchhoff-Love type) Consider a shell with middle surface $\boldsymbol{\theta}(\bar{\omega}) \subset \mathbb{E}^{3}$ and thickness $2 \varepsilon>0$ that satisfies the assumptions of Sect. 1. The Lamé constants of the elastic material constituting the shell being denoted by $\lambda>0$ and $\mu>0$, let

$$
\lambda^{*}:=\frac{2 \lambda \mu}{\lambda+2 \mu} .
$$

Define the set

$$
\begin{aligned}
& U_{\sharp}(\omega):=\left\{(\tilde{\boldsymbol{\theta}}, \tilde{\boldsymbol{\eta}}) \in W^{1,4}\left(\omega ; \mathbb{E}^{3}\right) \times W^{1,4}\left(\omega ; \mathbb{E}^{3}\right) ;|\tilde{\boldsymbol{\eta}}|=1 \text { in } \omega,\right. \\
&\left.\tilde{\boldsymbol{\eta}} \cdot \partial_{\alpha} \tilde{\boldsymbol{\theta}}=0 \text { a.e. in } \omega, \tilde{\boldsymbol{\theta}}=\boldsymbol{\theta} \text { on } \gamma_{0}, \tilde{\boldsymbol{\eta}}=\boldsymbol{a}_{3} \text { on } \gamma_{0}\right\},
\end{aligned}
$$

then the set

$$
\begin{array}{r}
U_{\sharp}\left(\Omega^{\varepsilon}\right):=\left\{\tilde{\boldsymbol{\Theta}} \in W^{1,4}\left(\Omega^{\varepsilon} ; \mathbb{E}^{3}\right) ; \exists(\tilde{\boldsymbol{\theta}}, \tilde{\boldsymbol{\eta}}) \in U_{\sharp}(\omega)\right. \text { such that } \\
\tilde{\boldsymbol{\Theta}}\left(y, x_{3}\right)=\tilde{\boldsymbol{\theta}}(y)+x_{3} \tilde{\boldsymbol{\eta}}(y) \text { for all }\left(y, x_{3}\right) \in \Omega^{\varepsilon}, \\
\left.\operatorname{det} \nabla \tilde{\boldsymbol{\Theta}}>0 \text { a.e. in } \Omega^{\varepsilon}\right\} .
\end{array}
$$

Given any $\tilde{\boldsymbol{\Theta}} \in U_{\sharp}\left(\Omega^{\varepsilon}\right)$, let

$$
\tilde{g}_{\alpha \beta}:=\partial_{\alpha} \tilde{\boldsymbol{\Theta}} \cdot \partial_{\beta} \tilde{\boldsymbol{\Theta}} \in L^{2}\left(\Omega^{\varepsilon}\right),
$$

let

$$
\begin{aligned}
W_{\sharp}^{\varepsilon}(\tilde{\mathbf{\Theta}}) & :=\frac{\mu}{8}\left(g^{\alpha \beta} \tilde{g}_{\alpha \beta}\right)^{2}+\left(\lambda^{*}-\mu\right) \sqrt{\operatorname{det}\left(g^{\alpha \sigma} \tilde{g}_{\sigma \beta}\right)} \\
& -\frac{\lambda^{*}}{2} \log \operatorname{det}\left(g^{\alpha \sigma} \tilde{g}_{\sigma \beta}\right)+\frac{\mu}{2}-\lambda^{*} \text { a.e. in } \Omega^{\varepsilon},
\end{aligned}
$$

and let

$$
J_{\sharp}^{\varepsilon}(\tilde{\mathbf{\Theta}}):=\int_{\Omega^{\varepsilon}} W_{\sharp}^{\varepsilon}(\tilde{\mathbf{\Theta}}) \sqrt{g} d x-\int_{\Omega^{\varepsilon}} \boldsymbol{f} \cdot \tilde{\mathbf{\Theta}} \sqrt{g} d x \in \mathbb{R} \cup\{+\infty\} .
$$

Then the unknown deformation $\Theta^{*}: \Omega^{\varepsilon} \rightarrow \mathbb{E}^{3}$ of the shell can be found by solving the minimization problem:

Find $\boldsymbol{\Theta}^{*} \in U_{\sharp}\left(\Omega^{\varepsilon}\right)$ such that $J_{\sharp}^{\varepsilon}\left(\mathbf{\Theta}^{*}\right) \leq J_{\sharp}^{\varepsilon}(\tilde{\boldsymbol{\Theta}})$ for all $\tilde{\boldsymbol{\Theta}} \in U_{\sharp}\left(\Omega^{\varepsilon}\right)$.

In what follows, we will first compare the above nonlinear shell model with the previous ones introduced in Definition 1 and Definition 2, then we will prove that it possesses at least a solution (Section 4).

Firstly, note that the nonlinear shell models of Definition 1 and Definition 3 share the same linear form modeling the applied forces.

Secondly, the minimization sets, $U\left(\Omega^{\varepsilon}\right)$ in Definition 1 and $U_{\sharp}\left(\Omega^{\varepsilon}\right)$ in Definition 3 , are not comparable, in the sense that, on the one hand,

$$
U\left(\Omega^{\varepsilon}\right) \not \subset U_{\sharp}\left(\Omega^{\varepsilon}\right)
$$


since a mapping $\tilde{\boldsymbol{\Theta}} \in U\left(\Omega^{\varepsilon}\right)$ is not necessarily orientation preserving at almost all points of $\Omega^{\varepsilon}$, and, on the other hand,

$$
U_{\sharp}\left(\Omega^{\varepsilon}\right) \not \subset U\left(\Omega^{\varepsilon}\right)
$$

since a mapping $\tilde{\boldsymbol{\Theta}} \in U_{\sharp}\left(\Omega^{\varepsilon}\right)$, which is of the form $\tilde{\boldsymbol{\Theta}}\left(y, x_{3}\right)=\tilde{\boldsymbol{\theta}}(y)+x_{3} \tilde{\boldsymbol{\eta}}(y)$ for all $\left(y, x_{3}\right) \in \Omega^{\varepsilon}$, does not necessarily satisfy $\tilde{\boldsymbol{\eta}}=\tilde{\boldsymbol{a}}_{3}$. However, note that both $\tilde{\boldsymbol{\eta}}$ and $\tilde{\boldsymbol{a}}_{3}$ are unit vector fields orthogonal to the vector fields $\tilde{\boldsymbol{a}}_{\alpha}:=\partial_{\alpha} \boldsymbol{\theta}$. Hence, if in addition $\tilde{\boldsymbol{\Theta}}$ is of class $\mathcal{C}^{1}$ (instead of only $W^{1,4}$ ), and if the assumption $\operatorname{det} \boldsymbol{\nabla} \tilde{\boldsymbol{\Theta}}>0$ is now satisfied at all points of $\Omega^{\varepsilon}$ (instead of only a.e. in $\left.\Omega^{\varepsilon}\right)$, then $\tilde{\boldsymbol{\eta}}=\tilde{\boldsymbol{a}}_{3}$.

Thirdly, the strain energy densities, $W^{\varepsilon}$ in Definition 1 and $W_{\sharp}^{\varepsilon}$ in Definition 3, coincide to within the first order with respect to the strain tensor field $\left(\tilde{E}_{\alpha \beta}\right)$, as we now show.

To begin with, we recall that the functions $\tilde{E}_{\alpha \beta}$ appearing in Definition 1 are related to the functions $\tilde{g}_{\alpha \beta}$ appearing in Definition 3 by

$$
\tilde{E}_{\alpha \beta}:=\frac{1}{2}\left(\tilde{g}_{\alpha \beta}-g_{\alpha \beta}\right) \text { a.e. in } \Omega^{\varepsilon} .
$$

Thus

$$
\tilde{g}_{\alpha \beta}=g_{\alpha \beta}+2 \tilde{E}_{\alpha \beta}
$$

which next implies that

$$
g^{\alpha \sigma} \tilde{g}_{\sigma \beta}=\delta_{\beta}^{\alpha}+2 g^{\alpha \sigma} \tilde{E}_{\sigma \beta},
$$

where $\delta_{\beta}^{\alpha}$ denotes Kronecker's symbol.

Under the assumption that the strain tensor field $\tilde{E}_{\alpha \beta}$ is sufficiently small (it suffices that $\left\|\left(g^{\alpha \sigma} \tilde{E}_{\sigma \beta}\right)\right\|<1 / 2$ a.e. in $\left.\Omega^{\varepsilon}\right)$, we deduce that

$$
\begin{aligned}
\operatorname{det}\left(g^{\alpha \sigma} \tilde{g}_{\sigma \beta}\right) & =\operatorname{det}\left(\delta_{\beta}^{\alpha}+2 g^{\alpha \sigma} \tilde{E}_{\sigma \beta}\right) \\
& =1+2 g^{\alpha \beta} \tilde{E}_{\alpha \beta}+4 \operatorname{det}\left(g^{\alpha \sigma} \tilde{E}_{\sigma \beta}\right) \\
& =1+2 g^{\alpha \beta} \tilde{E}_{\alpha \beta}+2\left(g^{\alpha \beta} \tilde{E}_{\alpha \beta}\right)^{2}-2 g^{\alpha \sigma} g^{\beta \tau} \tilde{E}_{\sigma \tau} \tilde{E}_{\alpha \beta}
\end{aligned}
$$

(the last equality is proved by using Cayley-Hamilton formula for the matrix field $\left.\left(g^{\alpha \sigma} \tilde{E}_{\sigma \beta}\right)\right)$. Consequently, by using the Taylor expansions $\sqrt{1+t}=1+$ $\frac{1}{2} t-\frac{1}{8} t^{2}+O\left(|t|^{3}\right)$ and $\log (1+t)=t-\frac{1}{2} t^{2}+O\left(|t|^{3}\right)$, we infer from the previous relation that

$\sqrt{\operatorname{det}\left(g^{\alpha \sigma} \tilde{g}_{\sigma \beta}\right)}=1+g^{\alpha \beta} \tilde{E}_{\alpha \beta}+\frac{1}{2}\left(g^{\alpha \beta} \tilde{E}_{\alpha \beta}\right)^{2}-g^{\alpha \sigma} g^{\beta \tau} \tilde{E}_{\sigma \tau} \tilde{E}_{\alpha \beta}+R_{1}\left(g^{\alpha \sigma} \tilde{E}_{\sigma \beta}\right)$

and

$$
\log \operatorname{det}\left(g^{\alpha \sigma} \tilde{g}_{\sigma \beta}\right)=2 g^{\alpha \beta} \tilde{E}_{\alpha \beta}-2 g^{\alpha \sigma} g^{\beta \tau} \tilde{E}_{\sigma \tau} \tilde{E}_{\alpha \beta}+R_{2}\left(g^{\alpha \sigma} \tilde{E}_{\sigma \beta}\right),
$$


where $R_{1}\left(g^{\alpha \sigma} \tilde{E}_{\sigma \beta}\right)=O\left(\left\|\left(g^{\alpha \sigma} \tilde{E}_{\sigma \beta}\right)\right\|^{3}\right)$ and $R_{2}\left(g^{\alpha \sigma} \tilde{E}_{\sigma \beta}\right)=O\left(\left\|\left(g^{\alpha \sigma} \tilde{E}_{\sigma \beta}\right)\right\|^{3}\right)$. Next, using the above relations in the definition of the stored energy function $W_{\sharp}^{\varepsilon}(\tilde{\boldsymbol{\Theta}})$ (Definition 3), we deduce that, a.e. in $\Omega^{\varepsilon}$,

$$
\begin{aligned}
W_{\sharp}^{\varepsilon}(\tilde{\boldsymbol{\Theta}}) & =\frac{\mu}{2}\left(1+g^{\alpha \beta} \tilde{E}_{\alpha \beta}\right)^{2} \\
& +\left(\lambda^{*}-\mu\right)\left(1+g^{\alpha \beta} \tilde{E}_{\alpha \beta}+\frac{1}{2}\left(g^{\alpha \beta} \tilde{E}_{\alpha \beta}\right)^{2}-g^{\alpha \sigma} g^{\beta \tau} \tilde{E}_{\sigma \tau} \tilde{E}_{\alpha \beta}\right) \\
& -\lambda^{*}\left(g^{\alpha \beta} \tilde{E}_{\alpha \beta}-g^{\alpha \sigma} g^{\beta \tau} \tilde{E}_{\sigma \tau} \tilde{E}_{\alpha \beta}\right)+\frac{\mu}{2}-\lambda^{*}+R_{3}\left(g^{\alpha \sigma} \tilde{E}_{\sigma \beta}\right),
\end{aligned}
$$

then that, using in particular the symmetry relations $\tilde{E}_{\alpha \beta}=\tilde{E}_{\beta \alpha}$,

$$
\begin{aligned}
W_{\sharp}^{\varepsilon}(\tilde{\boldsymbol{\Theta}}) & =\frac{\lambda^{*}}{2}\left(g^{\alpha \beta} \tilde{E}_{\alpha \beta}\right)^{2}+\mu g^{\alpha \sigma} g^{\beta \tau} \tilde{E}_{\sigma \tau} \tilde{E}_{\alpha \beta}+R_{3}\left(g^{\alpha \sigma} \tilde{E}_{\sigma \beta}\right) \\
& =\frac{1}{2}\left(\lambda^{*} g^{\alpha \beta} g^{\sigma \tau}+\mu\left(g^{\alpha \sigma} g^{\beta \tau}+g^{\alpha \tau} g^{\beta \sigma}\right)\right) \tilde{E}_{\sigma \tau} \tilde{E}_{\alpha \beta}+R_{3}\left(g^{\alpha \sigma} \tilde{E}_{\sigma \beta}\right) \\
& =\frac{1}{4} g^{\alpha \beta \sigma \tau} \tilde{E}_{\sigma \tau} \tilde{E}_{\alpha \beta}+R_{3}\left(g^{\alpha \sigma} \tilde{E}_{\sigma \beta}\right)
\end{aligned}
$$

where the tensor field $g^{\alpha \beta \sigma \tau}$ is precisely that defined in Definition 1 and

$$
R_{3}\left(g^{\alpha \sigma} \tilde{E}_{\sigma \beta}\right):=\left(\lambda^{*}-\mu\right) R_{1}\left(g^{\alpha \sigma} \tilde{E}_{\sigma \beta}\right)-\frac{\lambda^{*}}{2} R_{2}\left(g^{\alpha \sigma} \tilde{E}_{\sigma \beta}\right)=O\left(\left\|\left(g^{\alpha \sigma} \tilde{E}_{\sigma \beta}\right)\right\|^{3}\right) .
$$

Combined with the definition of the strain energy density $W^{\varepsilon}(\tilde{\boldsymbol{\Theta}})$ of the nonlinear shell model of Definition 1 , the above relation shows that

$$
\left|W_{\sharp}^{\varepsilon}(\tilde{\boldsymbol{\Theta}})-W^{\varepsilon}(\tilde{\boldsymbol{\Theta}})\right|=\left|R_{3}\left(g^{\alpha \sigma} \tilde{E}_{\sigma \beta}\right)\right|=O\left(\left\|\left(g^{\alpha \sigma} \tilde{E}_{\sigma \beta}\right)\right\|^{3}\right) .
$$

Hence the two nonlinear shell models of Definition 1 and Definition 3 coincide to within the first order with respect to the strain tensor field $\left(\tilde{E}_{\alpha \beta}\right)$ inside the shell, as claimed.

Finally, in order to compare the nonlinear shell model of Definition 3 with Koiter's nonlinear shell model (Definition 2), we assume in addition that $\tilde{\boldsymbol{\Theta}}$ is of class $\mathcal{C}^{1}$ and satisfies $\operatorname{det} \nabla \tilde{\boldsymbol{\Theta}}>0$ in $\Omega^{\varepsilon}$, so that $\tilde{\boldsymbol{\Theta}} \in U_{\sharp}\left(\Omega^{\varepsilon}\right)$ implies that $\tilde{\boldsymbol{\Theta}} \in U\left(\Omega^{\varepsilon}\right)$. Then we can use the estimate (12) between the strain energy densities in Definition 1 and Definition 2 to deduce from the above relation that

$$
\left|\int_{-\varepsilon}^{\varepsilon} W_{\sharp}^{\varepsilon}(\tilde{\boldsymbol{\Theta}}) \sqrt{g} d x_{3}-W_{K}^{\varepsilon}(\tilde{\boldsymbol{\theta}}) \sqrt{a}\right| \leq \int_{-\varepsilon}^{\varepsilon}\left|R_{3}\left(g^{\alpha \sigma} \tilde{E}_{\sigma \beta}\right)\right| \sqrt{g} d x_{3}+C_{2} \varepsilon W_{K}^{\varepsilon}(\tilde{\boldsymbol{\theta}}) \sqrt{a}
$$

for all $0<\varepsilon<\varepsilon_{0}$ and for all mappings $\tilde{\boldsymbol{\Theta}} \in U_{\sharp}\left(\Omega^{\varepsilon}\right) \cap U\left(\Omega^{\varepsilon}\right)$ that satisfy $\left\|\left(\tilde{b}_{\beta}^{\alpha}\right)\right\|_{L^{\infty}\left(\omega ; \mathbb{M}^{2}\right)} \leq M$ (the constant $C_{2}$ depends on $\varepsilon_{0}, \boldsymbol{\theta}$ and $\left.M\right)$. Furthermore, using that $\left|R_{3}\left(g^{\alpha \sigma} \tilde{E}_{\sigma \beta}\right)\right|=O\left(\left\|\left(g^{\alpha \sigma} \tilde{E}_{\sigma \beta}\right)\right\|^{3}\right)$, that

$$
\tilde{E}_{\alpha \beta}\left(\cdot, x_{3}\right)=\frac{1}{2}\left(\tilde{a}_{\alpha \beta}-a_{\alpha \beta}\right)-x_{3}\left(\tilde{b}_{\alpha \beta}-b_{\alpha \beta}\right)+\frac{x_{3}^{2}}{2}\left(\tilde{c}_{\alpha \beta}-c_{\alpha \beta}\right) \text { a.e. in } \omega
$$


for each $\left.x_{3} \in\right]-\varepsilon, \varepsilon[($ cf. Sect 2$)$, that

$$
\left\|\left(\tilde{c}_{\alpha \beta}-c_{\alpha \beta}\right)\right\| \leq C\left(\left\|\left(\tilde{b}_{\beta}^{\alpha}\right)\right\|\right)\left(\left\|\left(\tilde{a}_{\alpha \beta}-a_{\alpha \beta}\right)\right\|+\left\|\left(\tilde{b}_{\alpha \beta}-b_{\alpha \beta}\right)\right\|\right)
$$

(cf. (10)), and that the coerciveness inequality (11) holds for Koiter's strain energy density $W_{K}^{\varepsilon}(\tilde{\boldsymbol{\theta}})$, we deduce from the previous inequality that

$$
\left|\int_{-\varepsilon}^{\varepsilon} W_{\sharp}^{\varepsilon}(\tilde{\boldsymbol{\Theta}}) \sqrt{g} d x_{3}-W_{K}^{\varepsilon}(\tilde{\boldsymbol{\theta}}) \sqrt{a}\right| \leq C_{3} \varepsilon W_{K}^{\varepsilon}(\tilde{\boldsymbol{\theta}}) \sqrt{a},
$$

where the constant $C_{3}$ depends on $\varepsilon_{0}, \boldsymbol{\theta}$ and $M$.

We conclude this section by stating an existence theorem for the nonlinear shell model of Kirchhoff-Love type of Definition 3, the proof of which is given in the next section.

Theorem 1 Consider a shell satisfying the assumptions of Sect. 1. Then there exists a mapping $\Theta^{*}: \Omega^{\varepsilon} \rightarrow \mathbb{E}^{3}$ such that

$$
\boldsymbol{\Theta}^{*} \in U_{\sharp}\left(\Omega^{\varepsilon}\right) \text { and } J_{\sharp}^{\varepsilon}\left(\boldsymbol{\Theta}^{*}\right) \leq J_{\sharp}^{\varepsilon}(\tilde{\boldsymbol{\Theta}}) \text { for all } \tilde{\boldsymbol{\Theta}} \in U_{\sharp}\left(\Omega^{\varepsilon}\right) \text {. }
$$

\section{Proof of the existence theorem}

For clarity, the proof is broken into five sections, numbered Subsections 4.1 to 4.5. It is inspired by the proof of the existence theorem of John Ball for the three-dimensional model of nonlinear elasticity (Ref. [2]) and by the proof of the existence theorem of Ciarlet and Mardare for a specific nonlinear shell model with "two direction fields" (Ref. [9]).

\subsection{The functional $J_{\sharp}^{\varepsilon}: U_{\sharp}\left(\Omega^{\varepsilon}\right) \rightarrow \mathbb{R} \cup\{+\infty\}$ is well-defined}

Let $\left(g_{i j}\right) \in \mathcal{C}^{0}\left(\overline{\Omega^{\varepsilon}} ; \mathbb{S}_{>}^{3}\right)$ be the matrix field whose coefficients

$$
g_{i j}:=\partial_{i} \Theta \cdot \partial_{j} \Theta \text { in } \overline{\Omega^{\varepsilon}}
$$

are the covariant components of the metric tensor field associated with the immersion $\Theta \in \mathcal{C}^{1}\left(\overline{\Omega^{\varepsilon}} ; \mathbb{E}^{3}\right)$ defining the reference configuration of the shell (Sect. 1), and let $\left(g^{i j}(x)\right):=\left(g_{i j}(x)\right)^{-1} \in \mathbb{S}_{>}^{3}$ for all $x \in \overline{\Omega^{\varepsilon}}$. Then

$$
g_{\alpha 3}=g_{3 \alpha}=0 \text { and } g_{33}=1 \text { in } \overline{\Omega^{\varepsilon}},
$$

which next implies that the matrices $\left(g_{\alpha \beta}(x)\right) \in \mathbb{S}^{2}$ are positive-definite for all $x \in \overline{\Omega^{\varepsilon}}$, which in turn implies that

$$
\left(g^{\alpha \beta}(x)\right)=\left(g_{\alpha \beta}(x)\right)^{-1} \in \mathbb{S}_{>}^{2} \text { for all } x \in \overline{\Omega^{\varepsilon}} .
$$

Therefore the matrix

$$
\left(u^{\alpha \beta}(x):=\left(g^{\alpha \beta}(x)\right)^{1 / 2} \in \mathbb{S}_{>}^{2}\right.
$$


is well-defined, for all $x \in \overline{\Omega^{\varepsilon}}$ (the notation $A^{1 / 2}$ designates the square root of the matrix $A$; if $A$ is symmetric and positive definite, it is well-known that there exists a unique symmetric and positive-definite matrix $B$ such that $B^{2}=A$; hence $A^{1 / 2}:=B$ is well-defined).

Let $\tilde{\boldsymbol{\Theta}} \in U_{\sharp}\left(\Omega^{\varepsilon}\right)$ and let the matrix field $\left(\tilde{g}_{\alpha \beta}\right) \in L^{2}\left(\Omega^{\varepsilon} ; \mathbb{S}^{2}\right)$ be defined by

$$
\tilde{g}_{\alpha \beta}:=\partial_{\alpha} \tilde{\boldsymbol{\Theta}} \cdot \partial_{\beta} \tilde{\boldsymbol{\Theta}} \in L^{2}\left(\Omega^{\varepsilon}\right) .
$$

Then the matrix field

$$
\left(g^{\alpha \sigma} \tilde{g}_{\sigma \beta}\right) \in L^{2}\left(\Omega^{\varepsilon} ; \mathbb{M}^{2}\right)
$$

has the following properties a.e. in $\Omega^{\varepsilon}$ :

$$
\operatorname{det}\left(g^{\alpha \sigma} \tilde{g}_{\sigma \beta}\right)=\operatorname{det}\left(u^{\alpha \tau} u^{\tau \sigma} \tilde{g}_{\sigma \beta}\right)=\operatorname{det}\left(u^{\tau \sigma} \tilde{g}_{\sigma \beta} u^{\beta \alpha}\right) \geq 0,
$$

and

$$
g^{\alpha \beta} \tilde{g}_{\alpha \beta}=\operatorname{tr}\left(g^{\alpha \sigma} \tilde{g}_{\sigma \beta}\right)=\operatorname{tr}\left(u^{\alpha \tau} u^{\tau \sigma} \tilde{g}_{\sigma \beta}\right)=\operatorname{tr}\left(u^{\tau \sigma} \tilde{g}_{\sigma \beta} u^{\beta \alpha}\right) \geq 0 .
$$

Since, a.e. $x \in \Omega^{\varepsilon}$, the matrix $\left(u^{\alpha \sigma} \tilde{g}_{\sigma \tau} u^{\tau \beta}\right)(x)$ is symmetric and positive semi-definite, there exists a $2 \times 2$ orthogonal matrix $P(x)$ and a $2 \times 2$ diagonal matrix $D(x)$, with components $\lambda_{1}(x) \geq 0$ and $\lambda_{2}(x) \geq 0$ on its diagonal, such that

$$
\left(u^{\tau \sigma} \tilde{g}_{\sigma \beta} u^{\beta \alpha}\right)=P^{T} D P \text { a.e. in } \Omega^{\varepsilon} .
$$

We then infer from the previous three relations that, a.e. in $\Omega^{\varepsilon}$,

$$
\begin{aligned}
\operatorname{det}\left(g^{\alpha \sigma} \tilde{g}_{\sigma \beta}\right) & =\operatorname{det}\left(u^{\tau \sigma} \tilde{g}_{\sigma \beta} u^{\beta \alpha}\right)=\operatorname{det}\left(P^{T} D P\right)=\lambda_{1} \lambda_{2} \\
& \leq\left(\frac{\lambda_{1}+\lambda_{2}}{2}\right)^{2}=\frac{1}{4}\left(\operatorname{tr}\left(P^{T} D P\right)\right)^{2} \\
& =\frac{1}{4}\left(\operatorname{tr}\left(u^{\tau \sigma} \tilde{g}_{\sigma \beta} u^{\beta \alpha}\right)\right)^{2}=\frac{1}{4}\left(\operatorname{tr}\left(g^{\alpha \sigma} \tilde{g}_{\sigma \beta}\right)\right)^{2} \\
& =\frac{1}{4}\left(g^{\alpha \beta} \tilde{g}_{\alpha \beta}\right)^{2} .
\end{aligned}
$$

Therefore (recall that we have proved that $\operatorname{det}\left(g^{\alpha \sigma} \tilde{g}_{\sigma \beta}\right) \geq 0$ and $g^{\alpha \beta} \tilde{g}_{\alpha \beta} \geq 0$ a.e. in $\left.\Omega^{\varepsilon}\right)$ :

$$
\sqrt{\operatorname{det}\left(g^{\alpha \sigma} \tilde{g}_{\sigma \beta}\right)} \leq \frac{1}{2}\left(g^{\alpha \beta} \tilde{g}_{\alpha \beta}\right) \text { a.e. in } \Omega^{\varepsilon} .
$$

Besides, since $\log t \leq 2\left(t^{1 / 2}-1\right.$ ) for all $t \geq 0$ (the $\log$ function is extended at the origin by $\log 0:=-\infty$ ), we also have

$$
\log \operatorname{det}\left(g^{\alpha \sigma} \tilde{g}_{\sigma \beta}\right) \leq 2 \sqrt{\operatorname{det}\left(g^{\alpha \sigma} \tilde{g}_{\sigma \beta}\right)}-2 \leq g^{\alpha \beta} \tilde{g}_{\alpha \beta}-2 \text { a.e. in } \Omega^{\varepsilon} .
$$

Next, the inequalities above imply that the stored energy function $W_{\sharp}^{\varepsilon}(\tilde{\boldsymbol{\Theta}})$ defined in Definition 3 satisfies, a.e. in $\Omega^{\varepsilon}$,

$$
\begin{aligned}
W_{\sharp}^{\varepsilon}(\tilde{\boldsymbol{\Theta}}) & =\frac{\mu}{8}\left(g^{\alpha \beta} \tilde{g}_{\alpha \beta}\right)^{2}+\left(\lambda^{*}-\mu\right) \sqrt{\operatorname{det}\left(g^{\alpha \sigma} \tilde{g}_{\sigma \beta}\right)}-\frac{\lambda^{*}}{2} \log \operatorname{det}\left(g^{\alpha \sigma} \tilde{g}_{\sigma \beta}\right)+\frac{\mu}{2}-\lambda^{*} \\
& \geq \frac{\mu}{8}\left(g^{\alpha \beta} \tilde{g}_{\alpha \beta}\right)^{2}-\frac{\left|\lambda^{*}-\mu\right|}{2}\left(g^{\alpha \beta} \tilde{g}_{\alpha \beta}\right)-\frac{\lambda^{*}}{2}\left(g^{\alpha \beta} \tilde{g}_{\alpha \beta}-2\right)+\frac{\mu}{2}-\lambda^{*} \\
& \geq \frac{\mu}{8}\left(g^{\alpha \beta} \tilde{g}_{\alpha \beta}\right)^{2}-\left(\lambda^{*}+\frac{\mu}{2}\right)\left(g^{\alpha \beta} \tilde{g}_{\alpha \beta}\right)+\frac{\mu}{2},
\end{aligned}
$$


from which we deduce that $W_{\sharp}^{\varepsilon}(\tilde{\boldsymbol{\Theta}})$ satisfies the following inequality:

$$
W_{\sharp}^{\varepsilon}(\tilde{\mathbf{\Theta}}) \geq \frac{\mu}{9}\left(g^{\alpha \beta} \tilde{g}_{\alpha \beta}\right)^{2}-C_{0} \frac{1}{\sqrt{g}} \text { a.e. in } \Omega^{\varepsilon},
$$

where $C_{0}:=\frac{9 \mu}{2}\left(1+\frac{2 \lambda^{*}}{\mu}\right)^{2}-\frac{\mu}{2}$.

Since $g^{\alpha \beta} \in L^{\infty}\left(\Omega^{\varepsilon}\right)$ and $\sqrt{g} \in L^{\infty}\left(\Omega^{\varepsilon}\right)$ (thanks to the boundedness of the set $\omega$, which implies that $\Omega^{\varepsilon}$ is bounded too) and $\tilde{g}_{\alpha \beta} \in L^{2}\left(\Omega^{\varepsilon}\right)$, the above inequality implies that the (clearly measurable) function

$$
W_{\sharp}^{\varepsilon}(\tilde{\boldsymbol{\Theta}}) \sqrt{g}: \Omega^{\varepsilon} \rightarrow \mathbb{R} \cup\{+\infty\}
$$

is bounded from below by a function in $L^{1}\left(\Omega^{\varepsilon}\right)$; hence the integral

$$
\int_{\Omega^{\varepsilon}} W_{\sharp}^{\varepsilon}(\tilde{\mathbf{\Theta}}) \sqrt{g} d x \text { is well-defined in } \mathbb{R} \cup\{+\infty\} .
$$

Besides, since $\boldsymbol{f} \in L^{1}\left(\Omega^{\varepsilon} ; \mathbb{E}^{3}\right)$ and since the Sobolev embedding theorem implies that $\tilde{\boldsymbol{\Theta}} \in W^{1,4}\left(\Omega^{\varepsilon} ; \mathbb{E}^{3}\right) \subset L^{\infty}\left(\Omega^{\varepsilon} ; \mathbb{E}^{3}\right)$, the integral

$$
\int_{\Omega^{\varepsilon}} \boldsymbol{f} \cdot \tilde{\boldsymbol{\Theta}} \sqrt{g} d x \text { is well-defined in } \mathbb{R} .
$$

Hence the functional $J_{\sharp}^{\varepsilon}: U_{\sharp}\left(\Omega^{\varepsilon}\right) \rightarrow \mathbb{R} \cup\{+\infty\}$ is well defined.

\subsection{Coerciveness inequality for the functional $J_{\sharp}^{\varepsilon}$}

First, we infer from Sect. 4.1 that

$$
\int_{\Omega^{\varepsilon}} W_{\sharp}^{\varepsilon}(\tilde{\mathbf{\Theta}}) \sqrt{g} d x \geq \frac{\mu}{9} \int_{\Omega^{\varepsilon}}\left(g^{\alpha \beta} \tilde{g}_{\alpha \beta}\right)^{2} \sqrt{g} d x-C_{0}\left|\Omega^{\varepsilon}\right|
$$

and

$$
\left|\int_{\Omega^{\varepsilon}} \boldsymbol{f} \cdot \tilde{\boldsymbol{\Theta}} \sqrt{g} d x\right| \leq C_{1}\|\boldsymbol{f}\|_{L^{1}\left(\Omega^{\varepsilon} ; \mathbb{E}^{3}\right)}\|\tilde{\boldsymbol{\Theta}}\|_{W^{1,4}\left(\Omega^{\varepsilon} ; \mathbb{E}^{3}\right)},
$$

where $\left|\Omega^{\varepsilon}\right|$ denotes the Lebesgue measure of the set $\Omega^{\varepsilon} \subset \mathbb{R}^{3}$ and $C_{1}$ is a constant depending on $\boldsymbol{\theta}$ and on the norm of the Sobolev's embedding operator $W^{1,4}\left(\Omega^{\varepsilon} ; \mathbb{E}^{3}\right) \subset L^{\infty}\left(\Omega^{\varepsilon} ; \mathbb{E}^{3}\right)$. Moreover, since

$$
\begin{aligned}
\|\tilde{\boldsymbol{\Theta}}\|_{W^{1,4}\left(\Omega^{\varepsilon} ; \mathbb{E}^{3}\right)} & \leq\|\tilde{\boldsymbol{\theta}}\|_{W^{1,4}\left(\Omega^{\varepsilon} ; \mathbb{E}^{3}\right)}+\left\|x_{3} \tilde{\boldsymbol{\eta}}\right\|_{W^{1,4}\left(\Omega^{\varepsilon} ; \mathbb{E}^{3}\right)} \\
& \leq(2 \varepsilon)^{1 / 4}\left(\|\tilde{\boldsymbol{\theta}}\|_{W^{1,4}\left(\omega ; \mathbb{E}^{3}\right)}+\|\tilde{\boldsymbol{\eta}}\|_{L^{4}\left(\Omega^{\varepsilon} ; \mathbb{E}^{3}\right)}+\frac{\varepsilon}{\sqrt[4]{5}}\|\tilde{\boldsymbol{\eta}}\|_{W^{1,4}\left(\omega ; \mathbb{E}^{3}\right)}\right),
\end{aligned}
$$

we infer from the previous inequality that, for some constant $C_{2}(\varepsilon, \boldsymbol{f})$,

$$
\left|\int_{\Omega^{\varepsilon}} \boldsymbol{f} \cdot \tilde{\boldsymbol{\Theta}} \sqrt{g} d x\right| \leq C_{2}(\varepsilon, \boldsymbol{f})\left(\|\tilde{\boldsymbol{\theta}}\|_{W^{1,4}\left(\omega ; \mathbb{E}^{3}\right)}+\|\tilde{\boldsymbol{\eta}}\|_{W^{1,4}\left(\omega ; \mathbb{E}^{3}\right)}\right) .
$$


Therefore, combining inequalities (15) and (16) with the definition of the functional $J_{\sharp}^{\varepsilon}(\tilde{\boldsymbol{\Theta}})$ yields a first lower bound for this functional:

$$
\begin{aligned}
J_{\sharp}^{\varepsilon}(\tilde{\boldsymbol{\Theta}}) & =\int_{\Omega^{\varepsilon}} W_{\sharp}^{\varepsilon}(\tilde{\boldsymbol{\Theta}}) \sqrt{g} d x-\int_{\Omega^{\varepsilon}} \boldsymbol{f} \cdot \tilde{\boldsymbol{\Theta}} \sqrt{g} d x \\
& \geq \frac{\mu}{9} \int_{\Omega^{\varepsilon}}\left(g^{\alpha \beta} \tilde{g}_{\alpha \beta}\right)^{2} \sqrt{g} d x-C_{3}\left(\|\tilde{\boldsymbol{\theta}}\|_{W^{1,4}\left(\omega ; \mathbb{E}^{3}\right)}+\|\tilde{\boldsymbol{\eta}}\|_{W^{1,4}\left(\omega ; \mathbb{E}^{3}\right)}+1\right),
\end{aligned}
$$

where $C_{3}:=\max \left\{C_{0}\left|\Omega^{\varepsilon}\right|, C_{2}(\varepsilon, \boldsymbol{f})\right\}$.

Second, using that the matrix $\left(g^{\alpha \beta}(x)\right)$ is symmetric and positive-definite, we write

$$
\left(g^{\alpha \beta}\right)=P^{T} D P \text { in } \overline{\Omega^{\varepsilon}},
$$

where, for all $x \in \overline{\Omega^{\varepsilon}}, D(x)$ is a $2 \times 2$ diagonal matrix whose components on the diagonal are the two eigenvalues $0<\lambda_{\min }(x) \leq \lambda_{\max }(x)$ of the matrix $\left(g^{\alpha \beta}(x)\right)$, and $P(x)$ is a $2 \times 2$ orthogonal matrix. Then, for all $x \in \overline{\Omega^{\varepsilon}}$ and for all vectors $v_{\alpha}=\left(v_{\alpha}^{i}\right)_{i=1}^{3} \in \mathbb{R}^{3}, \alpha=1,2$, we have

$$
\begin{aligned}
g^{\alpha \beta}(x) v_{\alpha} \cdot v_{\beta} & =\sum_{i}\left(\sum_{\alpha, \beta} v_{\alpha}^{i} g^{\alpha \beta}(x) v_{\beta}^{i}\right) \\
& \geq \sum_{i}\left(\lambda_{\min }(x) \sum_{\alpha}\left(v_{\alpha}^{i}\right)^{2}\right)=\lambda_{\min }(x) \sum_{\alpha}\left|v_{\alpha}\right|^{2} .
\end{aligned}
$$

It follows that, for all $\tilde{\boldsymbol{\Theta}} \in U_{\sharp}\left(\Omega^{\varepsilon}\right)$,

$$
g^{\alpha \beta} \tilde{g}_{\alpha \beta}=g^{\alpha \beta} \partial_{\alpha} \tilde{\boldsymbol{\Theta}} \cdot \partial_{\beta} \tilde{\boldsymbol{\Theta}} \geq \sqrt{\lambda_{0}} \sum_{\alpha}\left|\partial_{\alpha} \tilde{\boldsymbol{\Theta}}\right|^{2} \text { a.e. in } \Omega^{\varepsilon}
$$

where $\lambda_{0}:=\inf _{x \in \overline{\Omega^{\varepsilon}}}\left(\lambda_{\min }(x)\right)^{2}>0$. Hence

$$
\begin{aligned}
\int_{\Omega^{\varepsilon}}\left(g^{\alpha \beta} \tilde{g}_{\alpha \beta}\right)^{2} d x & \geq \lambda_{0} \sum_{\alpha} \int_{\Omega^{\varepsilon}}\left|\partial_{\alpha} \tilde{\boldsymbol{\Theta}}\right|^{4} d x \\
& =\lambda_{0} \sum_{\alpha} \int_{\Omega^{\varepsilon}}\left|\partial_{\alpha} \tilde{\boldsymbol{\theta}}+x_{3} \partial_{\alpha} \tilde{\boldsymbol{\eta}}\right|^{4} d x
\end{aligned}
$$

Next, using the symmetry of the interval $]-\varepsilon, \varepsilon[$, Clarkson's inequality (see, e.g., [1, Theorem 2.28]), and Fubini integral formula, in the right hand side of the above inequality yields

$$
\begin{aligned}
\int_{\Omega^{\varepsilon}}\left(g^{\alpha \beta} \tilde{g}_{\alpha \beta}\right)^{2} d x & \geq \frac{\lambda_{0}}{2} \sum_{\alpha}\left(\int_{\Omega^{\varepsilon}}\left|\partial_{\alpha} \tilde{\boldsymbol{\theta}}+x_{3} \partial_{\alpha} \tilde{\boldsymbol{\eta}}\right|^{4} d x+\int_{\Omega^{\varepsilon}}\left|\partial_{\alpha} \tilde{\boldsymbol{\theta}}-x_{3} \partial_{\alpha} \tilde{\boldsymbol{\eta}}\right|^{4} d x\right) \\
& \geq \lambda_{0} \sum_{\alpha}\left(\int_{\Omega^{\varepsilon}}\left|\partial_{\alpha} \tilde{\boldsymbol{\theta}}\right|^{4} d x+\int_{\Omega^{\varepsilon}}\left|x_{3} \partial_{\alpha} \tilde{\boldsymbol{\eta}}\right|^{4} d x\right) \\
& =2 \lambda_{0} \sum_{\alpha}\left(\varepsilon \int_{\omega}\left|\partial_{\alpha} \tilde{\boldsymbol{\theta}}\right|^{4} d y+\frac{\varepsilon^{5}}{5} \int_{\omega}\left|\partial_{\alpha} \tilde{\boldsymbol{\eta}}\right|^{4} d y\right) \\
& =2 \lambda_{0}\left(\varepsilon\|\boldsymbol{\nabla} \tilde{\boldsymbol{\theta}}\|_{L^{4}\left(\omega ; \mathbb{M}^{3 \times 2}\right)}^{4}+\frac{\varepsilon^{5}}{5}\|\nabla \tilde{\boldsymbol{\eta}}\|_{L^{4}\left(\omega ; \mathbb{M}^{3 \times 2}\right)}^{4}\right) .
\end{aligned}
$$


Besides,

$$
\|\tilde{\boldsymbol{\eta}}\|_{L^{4}\left(\omega ; \mathbb{E}^{3}\right)}=\|1\|_{L^{4}\left(\omega ; \mathbb{E}^{3}\right)}=|\omega|^{1 / 4},
$$

and, since $\tilde{\boldsymbol{\theta}}=\boldsymbol{\theta}$ on $\gamma_{0}$, Poincaré inequality implies that (recall that $\omega$ is bounded and connected and that $\gamma_{0} \subset \partial \omega$ is relatively open and non-empty), for some constant $C_{4}<\infty$,

$$
\begin{aligned}
\|\tilde{\boldsymbol{\theta}}\|_{L^{4}\left(\omega ; \mathbb{E}^{3}\right)} & \leq\|\tilde{\boldsymbol{\theta}}-\boldsymbol{\theta}\|_{L^{4}\left(\omega ; \mathbb{E}^{3}\right)}+\|\boldsymbol{\theta}\|_{L^{4}\left(\omega ; \mathbb{E}^{3}\right)} \\
& \leq C_{4}\|\boldsymbol{\nabla} \tilde{\boldsymbol{\theta}}-\boldsymbol{\nabla} \boldsymbol{\theta}\|_{L^{4}\left(\omega ; \mathbb{M}^{3 \times 2}\right)}+\|\boldsymbol{\theta}\|_{L^{4}\left(\omega ; \mathbb{E}^{3}\right)} \\
& \leq C_{4}\|\boldsymbol{\nabla} \tilde{\boldsymbol{\theta}}\|_{L^{4}\left(\omega ; \mathbb{M}^{3 \times 2}\right)}+\left(1+C_{4}\right)\|\boldsymbol{\theta}\|_{W^{1,4}\left(\omega ; \mathbb{E}^{3}\right)}
\end{aligned}
$$

Then we infer from the last three inequalities that there exist two constants $C_{5}(\varepsilon, \boldsymbol{\theta})>0$ and $C_{6}(\varepsilon, \boldsymbol{\theta}) \in \mathbb{R}$ such that

$$
\int_{\Omega^{\varepsilon}}\left(g^{\alpha \beta} \tilde{g}_{\alpha \beta}\right)^{2} \sqrt{g} d x \geq C_{5}(\varepsilon)\left(\|\tilde{\boldsymbol{\theta}}\|_{W^{1,4}\left(\omega ; \mathbb{E}^{3}\right)}^{4}+\|\tilde{\boldsymbol{\eta}}\|_{W^{1,4}\left(\omega ; \mathbb{E}^{3}\right)}^{4}\right)-C_{6}(\varepsilon, \boldsymbol{\theta}) .
$$

Finally, using this inequality in the lower bound (17) established previously, we deduce that the functional $J_{\sharp}^{\varepsilon}(\tilde{\boldsymbol{\Theta}})$ is coercive in the sense that there exists two constants $A=A(\varepsilon, \mu, \boldsymbol{\theta})>0$ and $B=B(\varepsilon, \lambda, \mu, \boldsymbol{\theta}, \boldsymbol{f}) \in \mathbb{R}$ such that, for all $\tilde{\boldsymbol{\Theta}}\left(\cdot, x_{3}\right)=\tilde{\boldsymbol{\theta}}+x_{3} \tilde{\boldsymbol{\eta}} \in U_{\sharp}\left(\Omega^{\varepsilon}\right)$,

$$
J_{\sharp}^{\varepsilon}(\tilde{\boldsymbol{\Theta}}) \geq A\left(\|\tilde{\boldsymbol{\theta}}\|_{W^{1,4}\left(\omega ; \mathbb{E}^{3}\right)}^{4}+\|\tilde{\boldsymbol{\eta}}\|_{W^{1,4}\left(\omega ; \mathbb{E}^{3}\right)}^{4}\right)-B .
$$

\subsection{Convergence of an infimizing sequence}

Given any integer $k \geq 1$, pick any $\tilde{\boldsymbol{\Theta}}^{k} \in U_{\sharp}\left(\Omega^{\varepsilon}\right)$ that satisfies

$$
\inf _{\tilde{\boldsymbol{\Theta}} \in U_{\sharp}\left(\Omega^{\varepsilon}\right)} J_{\sharp}^{\varepsilon}(\tilde{\boldsymbol{\Theta}}) \leq J_{\sharp}^{\varepsilon}\left(\tilde{\boldsymbol{\Theta}}^{k}\right) \leq \inf _{\tilde{\boldsymbol{\Theta}} \in U_{\sharp}\left(\Omega^{\varepsilon}\right)} J_{\sharp}^{\varepsilon}(\tilde{\boldsymbol{\Theta}})+\frac{1}{k} .
$$

Since $\boldsymbol{\Theta} \in U_{\sharp}\left(\Omega^{\varepsilon}\right)$, we have

$$
\delta:=\inf _{\tilde{\boldsymbol{\Theta}} \in U_{\sharp}\left(\Omega^{\varepsilon}\right)} J_{\sharp}^{\varepsilon}(\tilde{\boldsymbol{\Theta}}) \leq J_{\sharp}^{\varepsilon}(\boldsymbol{\Theta})=-\int_{\Omega^{\varepsilon}} \boldsymbol{f} \cdot \boldsymbol{\Theta} \sqrt{g} d x<\infty .
$$

Since $\tilde{\boldsymbol{\Theta}}^{k} \in U_{\sharp}\left(\Omega^{\varepsilon}\right)$, there exist vector fields $\left(\tilde{\boldsymbol{\theta}}^{k}, \tilde{\boldsymbol{\eta}}^{k}\right) \in U_{\sharp}(\omega)$ such that

$$
\tilde{\boldsymbol{\Theta}}^{k}\left(y, x_{3}\right)=\tilde{\boldsymbol{\theta}}^{k}(y)+x_{3} \tilde{\boldsymbol{\eta}}^{k}(y) \text { for all }\left(y, x_{3}\right) \in \Omega^{\varepsilon} \text {. }
$$

Then the coerciveness inequality (19) implies that $\delta \in \mathbb{R}$ and that both sequences $\left(\tilde{\boldsymbol{\theta}}^{k}\right)_{k \geq 1}$ and $\left(\tilde{\boldsymbol{\eta}}^{k}\right)_{k \geq 1}$ are bounded in $W^{1,4}\left(\omega ; \mathbb{E}^{3}\right)$. Therefore there exist vector fields

$$
\boldsymbol{\theta}^{*} \in W^{1,4}\left(\omega ; \mathbb{E}^{3}\right) \text { and } \boldsymbol{\eta}^{*} \in W^{1,4}\left(\omega ; \mathbb{E}^{3}\right)
$$

such that

$$
\tilde{\boldsymbol{\theta}}^{k} \rightarrow \boldsymbol{\theta}^{*} \text { and } \tilde{\boldsymbol{\eta}}^{k} \rightarrow \boldsymbol{\eta}^{*} \text { in } W^{1,4}\left(\omega ; \mathbb{E}^{3}\right) \text { as } k \rightarrow \infty
$$


Since $W^{1,4}\left(\omega ; \mathbb{E}^{3}\right) \subset \mathcal{C}^{0}\left(\bar{\omega} ; \mathbb{E}^{3}\right)$ by Sobolev's embedding theorem, it follows that

$$
\tilde{\boldsymbol{\theta}}^{k} \rightarrow \boldsymbol{\theta}^{*} \text { and } \tilde{\boldsymbol{\eta}}^{k} \rightarrow \boldsymbol{\eta}^{*} \text { in } \mathcal{C}^{0}\left(\bar{\omega} ; \mathbb{E}^{3}\right) \text { as } k \rightarrow \infty \text {. }
$$

This implies in particular that $\boldsymbol{\theta}^{*}=\boldsymbol{\theta}$ and $\boldsymbol{\eta}^{*}=\boldsymbol{a}_{3}$ on $\gamma_{0}$.

Note also that the relations

$$
\left|\tilde{\boldsymbol{\eta}}^{k}\right|=1 \text { in } \omega \text { and } \tilde{\boldsymbol{\eta}}^{k} \cdot \partial_{\alpha} \tilde{\boldsymbol{\theta}}^{k}=0 \text { a.e. in } \omega,
$$

combined with the above weak and strong convergences of these sequences, imply that

$$
\left|\boldsymbol{\eta}^{*}\right|=1 \text { in } \omega \text { and } \boldsymbol{\eta}^{*} \cdot \partial_{\alpha} \boldsymbol{\theta}^{*}=0 \text { a.e. in } \omega \text {. }
$$

Hence $\left(\boldsymbol{\theta}^{*}, \boldsymbol{\eta}^{*}\right) \in U_{\sharp}(\omega)$ and the function $\boldsymbol{\Theta}^{*}: \Omega^{\varepsilon} \rightarrow \mathbb{E}^{3}$, defined by

$$
\boldsymbol{\Theta}^{*}(x)=\boldsymbol{\theta}^{*}(y)+x_{3} \boldsymbol{\eta}^{*}(y) \text { for all } x=\left(y, x_{3}\right) \in \Omega^{\varepsilon},
$$

belongs to the space $W^{1,4}\left(\Omega^{\varepsilon} ; \mathbb{E}^{3}\right)$.

Clearly, the sequence $\left(\tilde{\boldsymbol{\Theta}}^{k}\right)_{k \geq 1}$ satisfies

$$
\tilde{\boldsymbol{\Theta}}^{k} \rightarrow \boldsymbol{\Theta}^{*} \text { in } W^{1,4}\left(\Omega^{\varepsilon} ; \mathbb{E}^{3}\right) \text { as } k \rightarrow \infty,
$$

and

$$
\tilde{\boldsymbol{\Theta}}^{k} \rightarrow \boldsymbol{\Theta}^{*} \text { in } \mathcal{C}^{0}\left(\overline{\Omega^{\varepsilon}} ; \mathbb{E}^{3}\right) \text { as } k \rightarrow \infty
$$

\subsection{The limit of the infimizing sequence belongs to $U_{\sharp}\left(\Omega^{\varepsilon}\right)$}

Let $\boldsymbol{v}=\left(v_{i}\right)_{i=1}^{3} \in \mathcal{C}_{c}^{1}\left(\Omega^{\varepsilon} ; \mathbb{E}^{3}\right)$ denote any vector field whose components $v_{i}$ belong to $\mathcal{C}^{1}\left(\Omega^{\varepsilon}\right)$ and have compact support contained in $\Omega^{\varepsilon}$. Then

$\int_{\Omega^{\varepsilon}}\left(\partial_{1} \tilde{\boldsymbol{\Theta}}^{k} \wedge \partial_{2} \tilde{\boldsymbol{\Theta}}^{k}\right) \cdot \boldsymbol{v} d x=-\frac{1}{2} \int_{\Omega^{\varepsilon}}\left(\left(\tilde{\boldsymbol{\Theta}}^{k} \wedge \partial_{2} \tilde{\boldsymbol{\Theta}}^{k}\right) \cdot \partial_{1} \boldsymbol{v}+\left(\partial_{1} \tilde{\boldsymbol{\Theta}}^{k} \wedge \tilde{\boldsymbol{\Theta}}^{k}\right) \cdot \partial_{2} \boldsymbol{v}\right) d x$

and

$\int_{\Omega^{\varepsilon}}\left(\partial_{1} \boldsymbol{\Theta}^{*} \wedge \partial_{2} \boldsymbol{\Theta}^{*}\right) \cdot \boldsymbol{v} d x=-\frac{1}{2} \int_{\Omega^{\varepsilon}}\left(\left(\boldsymbol{\Theta}^{*} \wedge \partial_{2} \boldsymbol{\Theta}^{*}\right) \cdot \partial_{1} \boldsymbol{v}+\left(\partial_{1} \boldsymbol{\Theta}^{*} \wedge \boldsymbol{\Theta}^{*}\right) \cdot \partial_{2} \boldsymbol{v}\right) d x$

Since $\tilde{\boldsymbol{\Theta}}^{k} \rightarrow \boldsymbol{\Theta}^{*}$ in $W^{1,4}\left(\Omega^{\varepsilon} ; \mathbb{E}^{3}\right)$ and $\tilde{\boldsymbol{\Theta}}^{k} \rightarrow \boldsymbol{\Theta}^{*}$ in $\mathcal{C}^{0}\left(\overline{\Omega^{\varepsilon}} ; \mathbb{E}^{3}\right)$ (cf. Subsection 4.2$)$ the above relations imply that

$$
\lim _{k \rightarrow \infty} \int_{\Omega^{\varepsilon}}\left(\partial_{1} \tilde{\boldsymbol{\Theta}}^{k} \wedge \partial_{2} \tilde{\boldsymbol{\Theta}}^{k}\right) \cdot \boldsymbol{v} d x=\int_{\Omega^{\varepsilon}}\left(\partial_{1} \boldsymbol{\Theta}^{*} \wedge \partial_{2} \mathbf{\Theta}^{*}\right) \cdot \boldsymbol{v} d x
$$

Since $\mathcal{C}_{c}^{1}\left(\Omega^{\varepsilon} ; \mathbb{E}^{3}\right)$ is dense in $L^{2}\left(\Omega^{\varepsilon} ; \mathbb{E}^{3}\right)$ and the sequence $\left(\partial_{1} \tilde{\boldsymbol{\Theta}}^{k} \wedge \partial_{2} \tilde{\boldsymbol{\Theta}}^{k}\right)_{k \geq 1}$ is bounded in $L^{2}\left(\Omega ; \mathbb{E}^{3}\right)$, the above convergence implies that

$$
\partial_{1} \tilde{\boldsymbol{\Theta}}^{k} \wedge \partial_{2} \tilde{\boldsymbol{\Theta}}^{k} \rightarrow \partial_{1} \boldsymbol{\Theta}^{*} \wedge \partial_{2} \boldsymbol{\Theta}^{*} \text { in } L^{2}\left(\Omega ; \mathbb{E}^{3}\right),
$$

on the one hand. 
Since, on the other hand,

$$
\partial_{3} \tilde{\boldsymbol{\Theta}}^{k}=\tilde{\boldsymbol{\eta}}^{k} \rightarrow \boldsymbol{\eta}^{*}=\partial_{3} \boldsymbol{\Theta}^{*} \text { in } \mathcal{C}^{0}\left(\overline{\Omega^{\varepsilon}} ; \mathbb{E}^{3}\right),
$$

we conclude that

$$
\left(\partial_{1} \tilde{\boldsymbol{\Theta}}^{k} \wedge \partial_{2} \tilde{\mathbf{\Theta}}^{k}\right) \cdot \partial_{3} \tilde{\boldsymbol{\Theta}}^{k} \rightarrow\left(\partial_{1} \boldsymbol{\Theta}^{*} \wedge \partial_{2} \boldsymbol{\Theta}^{*}\right) \cdot \partial_{3} \boldsymbol{\Theta}^{*} \text { in } L^{2}(\Omega),
$$

or equivalently, that

$$
\operatorname{det} \nabla \tilde{\Theta}^{k} \rightarrow \operatorname{det} \nabla \Theta^{*} \text { in } L^{2}(\Omega) .
$$

Next, since $\tilde{\boldsymbol{\Theta}}^{k} \in U_{\sharp}\left(\Omega^{\varepsilon}\right)$, we have (cf. relation (13) in Section 3)

$$
\operatorname{det}\left(\tilde{g}_{\alpha \beta}^{k}\right)=\left(\operatorname{det} \nabla \tilde{\boldsymbol{\Theta}}^{k}\right)^{2} \text { a.e. in } \Omega^{\varepsilon} .
$$

Since the mapping $\tilde{\boldsymbol{\Theta}}^{k}$ satisfies in addition (cf. definition of the set $U_{\sharp}\left(\Omega^{\varepsilon}\right)$ )

$$
\operatorname{det} \nabla \tilde{\Theta}^{k}>0 \text { a.e. in } \Omega^{\varepsilon},
$$

we deduce from the previous relation that

$$
\sqrt{\operatorname{det}\left(\tilde{g}_{\alpha \beta}^{k}\right)}=\operatorname{det} \nabla \tilde{\Theta}^{k} \text { a.e. in } \Omega^{\varepsilon} .
$$

Then we infer from relations (20) and (22) that

$$
\sqrt{\operatorname{det}\left(\tilde{g}_{\alpha \beta}^{k}\right)}=\operatorname{det} \nabla \tilde{\Theta}^{k} \rightarrow \operatorname{det} \nabla \Theta^{*} \text { in } L^{2}(\Omega),
$$

and that

$$
\operatorname{det} \nabla \Theta^{*} \geq 0 \text { a.e. in } \Omega^{\varepsilon} .
$$

Next we prove that the following stronger inequality holds:

$$
\operatorname{det} \nabla \Theta^{*}>0 \text { a.e. in } \Omega^{\varepsilon} \text {. }
$$

Assume on the contrary that there exists a subset $A \subset \Omega^{\varepsilon}$ with positive Lebesque measure such that $\operatorname{det} \nabla \Theta^{*}=0$ a.e. in $A$. Then (20) and (21) imply that

$$
\operatorname{det} \boldsymbol{\nabla} \tilde{\boldsymbol{\Theta}}^{k} \rightarrow 0 \text { in } L^{2}(A) \text { and } \operatorname{det} \boldsymbol{\nabla} \tilde{\boldsymbol{\Theta}}^{k}>0 \text { a.e. in } A .
$$

In particular,

$$
\left\|\operatorname{det} \boldsymbol{\nabla} \tilde{\boldsymbol{\Theta}}^{k}\right\|_{L^{1}(A)}=\int_{A} \operatorname{det} \nabla \tilde{\boldsymbol{\Theta}}^{k} d x \rightarrow 0 \text { as } k \rightarrow \infty,
$$

which in turn implies the existence of a subsequence $(\ell)$ of $(k)$ such that

$$
\operatorname{det} \nabla \tilde{\Theta}^{\ell} \rightarrow 0 \text { a.e. in } A .
$$


Using Fatou's lemma, we next deduce that $\left(C_{0}\right.$ is the constant appearing in the coerciveness inequality (14)), on the one hand,

$$
\begin{array}{r}
\liminf _{\ell \rightarrow \infty} \int_{\Omega^{\varepsilon}}\left(W_{\sharp}^{\varepsilon}\left(\tilde{\boldsymbol{\Theta}}^{\ell}\right) \sqrt{g}+C_{0}\right) d x \geq \int_{\Omega^{\varepsilon}} \liminf _{\ell \rightarrow \infty}\left(W_{\sharp}^{\varepsilon}\left(\tilde{\boldsymbol{\Theta}}^{\ell}\right) \sqrt{g}+C_{0}\right) d x \\
\geq \int_{A} \liminf _{\ell \rightarrow \infty}\left(W_{\sharp}^{\varepsilon}\left(\tilde{\boldsymbol{\Theta}}^{\ell}\right) \sqrt{g}+C_{0}\right) d x=+\infty,
\end{array}
$$

the last equality being proved as follows (recall in particular that $\lambda^{*}>0$ ):

$$
\begin{aligned}
W_{\sharp}^{\varepsilon}\left(\tilde{\boldsymbol{\Theta}}^{\ell}\right) & \geq\left(\lambda^{*}-\mu\right) \sqrt{\operatorname{det}\left(g^{\alpha \sigma} \tilde{g}_{\sigma \beta}^{\ell}\right)}-\frac{\lambda^{*}}{2} \log \operatorname{det}\left(g^{\alpha \sigma} \tilde{g}_{\sigma \beta}^{\ell}\right)+\frac{\mu}{2}-\lambda^{*} \\
& =\frac{\left(\lambda^{*}-\mu\right)}{\sqrt{g}} \operatorname{det} \nabla \tilde{\Theta}^{\ell}-\lambda^{*} \log \left(\operatorname{det} \nabla \tilde{\Theta}^{\ell}\right)+\frac{\lambda^{*}}{2} \log (\operatorname{det} g)+\frac{\mu}{2}-\lambda^{*} \\
& \rightarrow+\infty \text { a.e. in } A .
\end{aligned}
$$

But, on the other hand,

$$
\begin{aligned}
\int_{\Omega^{\varepsilon}}\left(W_{\sharp}^{\varepsilon}\left(\tilde{\boldsymbol{\Theta}}^{\ell}\right) \sqrt{g}+C_{0}\right) d x & =J_{\sharp}^{\varepsilon}\left(\tilde{\boldsymbol{\Theta}}^{\ell}\right)+\int_{\Omega^{\varepsilon}} \boldsymbol{f} \cdot \tilde{\boldsymbol{\Theta}}^{\ell} \sqrt{g} d x+C_{0} \int_{\Omega^{\varepsilon}} d x \\
& \rightarrow \delta+\int_{\Omega^{\varepsilon}} \boldsymbol{f} \cdot \boldsymbol{\Theta}^{*} \sqrt{g} d x+C_{0} \int_{\Omega^{\varepsilon}} d x<\infty
\end{aligned}
$$

(that $\delta:=\inf _{\tilde{\boldsymbol{\Theta}} \in U_{\sharp}\left(\Omega^{\varepsilon}\right)} J_{\sharp}^{\varepsilon}(\tilde{\boldsymbol{\Theta}})<\infty$ is proved in Sect. 4.3).

This contradiction shows that we have indeed

$$
\operatorname{det} \nabla \Theta^{*}>0 \text { a.e. in } \Omega^{\varepsilon} \text {. }
$$

Hence $\boldsymbol{\Theta}^{*} \in U_{\sharp}\left(\Omega^{\varepsilon}\right)$.

\subsection{The inequality $\liminf \operatorname{in}_{k \rightarrow \infty} J_{\sharp}^{\varepsilon}\left(\tilde{\Theta}^{k}\right) \geq J_{\sharp}^{\varepsilon}\left(\boldsymbol{\Theta}^{*}\right)$ holds}

Define the function $G:[0, \infty[\rightarrow \mathbb{R} \cup\{+\infty\}$ by letting

$$
\begin{aligned}
& G(t)=\left(\lambda^{*}-\mu\right) t-\lambda^{*} \log t+\left(\frac{\mu}{2}-\lambda^{*}\right) \text { if } t>0, \\
& G(t)=+\infty \text { if } t=0 .
\end{aligned}
$$

Then we infer from the definition of the strain energy density $W_{\sharp}^{\varepsilon}$ (Definition 3) that

$$
W_{\sharp}^{\varepsilon}\left(\tilde{\boldsymbol{\Theta}}^{k}\right)=\frac{\mu}{8}\left(g^{\alpha \beta} \tilde{g}_{\alpha \beta}^{k}\right)^{2}+G\left(\sqrt{\operatorname{det}\left(g^{\alpha \sigma} \tilde{g}_{\sigma \beta}^{k}\right)}\right) .
$$

We have seen previously (Subsections 4.1 and 4.2) that

$$
g^{\alpha \beta} \tilde{g}_{\alpha \beta}^{k}=g^{\alpha \beta} \partial_{\alpha} \tilde{\boldsymbol{\Theta}}^{k} \cdot \partial_{\beta} \tilde{\boldsymbol{\Theta}}^{k} \text { in } L^{2}\left(\Omega^{\varepsilon}\right),
$$

and that, for all $x \in \overline{\Omega^{\varepsilon}}$, the matrix $\left(g^{\alpha \beta}(x)\right)$ is symmetric and positive definite and there exists a constant $\lambda_{0}>0$ such that

$$
g^{\alpha \beta}(x) v_{\alpha} \cdot v_{\beta} \geq \sqrt{\lambda_{0}} \sum_{\alpha}\left|v_{\alpha}\right|^{2}
$$


for all $x \in \overline{\Omega^{\varepsilon}}$ and all vectors $v_{\alpha}=\left(v_{\alpha}^{i}\right)_{i=1}^{3} \in \mathbb{R}^{3}, \alpha=1,2$ (cf. (18)). It follows that, for each $x \in \overline{\Omega^{\varepsilon}}$, the function

$$
F=\left(F_{\alpha}^{i}\right) \in \mathbb{M}^{3 \times 2} \mapsto\|F\|:=\left(\left(\sum_{\alpha, \beta, i} g^{\alpha \beta}(x) F_{\alpha}^{i} F_{\beta}^{i}\right)^{2}\right)^{1 / 4} \in \mathbb{R}
$$

is a norm in the space $\mathbb{M}^{3 \times 2}$, which is in addition uniformly equivalent with the Frobenius norm in the following sense: there exists two constants $C_{7}$ and $C_{8}$ depending only on $\boldsymbol{\theta}$ (independent of $x$ in particular) such that

$$
C_{7}\|F\| \leq\|F\| \leq C_{8}\|F\| \text { for all } F \in \mathbb{M}^{3 \times 2} .
$$

Therefore, $g^{\alpha \beta} \tilde{g}_{\alpha \beta}^{k} \geq 0$ a.e. in $\Omega^{\varepsilon}, \sqrt{g^{\alpha \beta} \tilde{g}_{\alpha \beta}^{k}} \in L^{4}\left(\Omega^{\varepsilon}\right)$, and

$$
\left(g^{\alpha \beta} \tilde{g}_{\alpha \beta}^{k}\right)^{2}=\left(g^{\alpha \beta} \partial_{\alpha} \tilde{\boldsymbol{\Theta}}^{k} \cdot \partial_{\beta} \tilde{\boldsymbol{\Theta}}^{k}\right)^{2}=\left.\left\|\nabla_{H} \tilde{\boldsymbol{\Theta}}^{k}\right\|\right|^{4},
$$

where the notation

$$
\nabla_{H} \tilde{\boldsymbol{\Theta}}^{k}:=\left(\partial_{1} \tilde{\mathbf{\Theta}}^{k} \mid \partial_{2} \tilde{\mathbf{\Theta}}^{k}\right) \in L^{4}\left(\Omega^{\varepsilon} ; \mathbb{M}^{3 \times 2}\right)
$$

designates the $(3 \times 2)$-matrix field $\boldsymbol{\nabla}_{H} \tilde{\boldsymbol{\Theta}}^{k}$ whose columns are the $(3 \times 1)$-vector fields $\partial_{1} \tilde{\boldsymbol{\Theta}}^{k}$ and $\partial_{2} \tilde{\boldsymbol{\Theta}}^{k}$.

Next, we have (cf. relation (22))

$$
\sqrt{\operatorname{det}\left(\tilde{g}_{\alpha \beta}^{k}\right)}=\operatorname{det} \nabla \tilde{\Theta}^{k} \text { a.e. in } \Omega^{\varepsilon},
$$

which in turn implies that

$$
\sqrt{\operatorname{det}\left(g^{\alpha \sigma} \tilde{g}_{\sigma \beta}^{k}\right)}=\frac{\sqrt{\operatorname{det}\left(\tilde{g}_{\alpha \beta}^{k}\right)}}{\sqrt{\operatorname{det}\left(g_{\alpha \beta}\right)}}=\frac{1}{\sqrt{g}} \operatorname{det} \nabla \tilde{\mathbf{\Theta}}^{k} \text { a.e. in } \Omega^{\varepsilon} \text {. }
$$

Using the above expressions of $\left(g^{\alpha \beta} \tilde{g}_{\alpha \beta}^{k}\right)^{2}$ and $\sqrt{\operatorname{det}\left(g^{\alpha \sigma} \tilde{g}_{\sigma \beta}^{k}\right)}$ in formula (24) of $W_{\sharp}^{\varepsilon}$ shows that

$$
W_{\sharp}^{\varepsilon}\left(\tilde{\mathbf{\Theta}}^{k}\right)=\frac{\mu}{8}\left\|\mid \nabla_{H} \tilde{\boldsymbol{\Theta}}^{k}\right\|^{4}+G\left(\frac{1}{\sqrt{g}} \operatorname{det}\left(\boldsymbol{\nabla} \tilde{\mathbf{\Theta}}^{k}\right)\right) .
$$

The weak convergences (see in particular (20))

$$
\boldsymbol{\nabla}_{H} \tilde{\boldsymbol{\Theta}}^{k} \rightarrow \boldsymbol{\nabla}_{H} \boldsymbol{\Theta}^{*} \text { in } L^{4}\left(\Omega^{\varepsilon} ; \mathbb{M}^{3 \times 2}\right) \text { and } \operatorname{det} \boldsymbol{\nabla} \tilde{\Theta}^{k} \rightarrow \operatorname{det} \nabla \Theta^{*} \text { in } L^{2}(\Omega),
$$

combined with the convexity of the functions $\|\cdot\| \|^{4}$ and $G$, and with the lower bound (14) for the function $W_{\sharp}^{\varepsilon}(\tilde{\mathbf{\Theta}})$, imply by means of a classical theorem in calculus of variations (see, e.g., Ball, Currie and Olver [3, Theorem 5.4] or Bunoiu, Ciarlet and Mardare [4, Theorem 1]) that

$$
\liminf _{k \rightarrow \infty} \int_{\Omega^{\varepsilon}} W_{\sharp}^{\varepsilon}\left(\tilde{\Theta}^{k}\right) \sqrt{g} d x \geq \int_{\Omega^{\varepsilon}}\left(\frac{\mu}{8}\left\|\left|\nabla_{H} \boldsymbol{\Theta}^{*} \|\right|^{4}+G\left(\frac{1}{\sqrt{g}} \operatorname{det} \boldsymbol{\nabla} \boldsymbol{\Theta}^{*}\right)\right) \sqrt{g} d x .\right.
$$


The integrand in the right-hand side can be recast as follows. Firstly, as in (25), we have

$$
\left\|\boldsymbol{\nabla}_{H} \boldsymbol{\Theta}^{*}\right\|^{4}=\left(g^{\alpha \beta} g_{\alpha \beta}^{*}\right)^{2} \text { a.e. in } \Omega^{\varepsilon} .
$$

Secondly, since $\boldsymbol{\Theta}^{*} \in U_{\sharp}\left(\Omega^{\varepsilon}\right)$, we also have (cf. relation (13) in Sect. 3)

$$
\left(\operatorname{det} \nabla \Theta^{*}\right)^{2}=\operatorname{det}\left(g_{\alpha \beta}^{*}\right) \text { a.e. in } \Omega^{\varepsilon} \text {, }
$$

which combined with the inequality $\operatorname{det}\left(g_{\alpha \beta}^{*}\right)>0$ a.e. in $\Omega^{\varepsilon}$ (this has been proven in the previous Subsection; cf. (23)) shows that

$$
\operatorname{det} \nabla \Theta^{*}=\sqrt{\operatorname{det}\left(g_{\alpha \beta}^{*}\right)} \text { a.e. in } \Omega^{\varepsilon} \text {. }
$$

Consequently,

$$
\frac{1}{\sqrt{g}} \operatorname{det} \nabla \Theta^{*}=\frac{\sqrt{\operatorname{det}\left(g_{\alpha \beta}^{*}\right)}}{\sqrt{\operatorname{det}\left(g_{\alpha \beta}\right)}}=\sqrt{\operatorname{det}\left(g^{\alpha \sigma} g_{\sigma \beta}^{*}\right)} \text { a.e. in } \Omega^{\varepsilon} \text {. }
$$

Then we infer from the previous inequality that

$$
\begin{aligned}
\liminf _{k \rightarrow \infty} \int_{\Omega^{\varepsilon}} W_{\sharp}^{\varepsilon}\left(\tilde{\boldsymbol{\Theta}}^{k}\right) \sqrt{g} d x & \geq \int_{\Omega^{\varepsilon}}\left(\frac{\mu}{8}\left(g^{\alpha \beta} g_{\alpha \beta}^{*}\right)^{2}+G\left(\sqrt{\operatorname{det}\left(g^{\alpha \sigma} g_{\alpha \beta}^{*}\right)}\right)\right) \sqrt{g} d x \\
& =\int_{\Omega^{\varepsilon}} W_{\sharp}^{\varepsilon}\left(\mathbf{\Theta}^{*}\right) \sqrt{g} d x .
\end{aligned}
$$

Besides, the strong convergence $\tilde{\boldsymbol{\Theta}}^{k} \rightarrow \boldsymbol{\Theta}^{*}$ in $\mathcal{C}^{0}\left(\overline{\Omega^{\varepsilon}} ; \mathbb{E}^{3}\right)$ implies that

$$
\lim _{k \rightarrow \infty} \int_{\Omega^{\varepsilon}} \boldsymbol{f} \cdot \tilde{\boldsymbol{\Theta}}^{k} \sqrt{g} d x=\int_{\Omega^{\varepsilon}} \boldsymbol{f} \cdot \boldsymbol{\Theta}^{*} \sqrt{g} d x .
$$

Finally, by combining the two limits above, we deduce that

$$
\begin{aligned}
J_{\sharp}^{\varepsilon}\left(\boldsymbol{\Theta}^{*}\right) & =\int_{\Omega^{\varepsilon}} W_{\sharp}^{\varepsilon}\left(\boldsymbol{\Theta}^{*}\right) \sqrt{g} d x-\int_{\Omega^{\varepsilon}} \boldsymbol{f} \cdot \boldsymbol{\Theta}^{*} \sqrt{g} d x \\
& \leq \liminf _{k \rightarrow \infty} J_{\sharp}^{\varepsilon}\left(\tilde{\boldsymbol{\Theta}}^{k}\right)=\inf _{\tilde{\boldsymbol{\Theta}} \in U_{\sharp}\left(\Omega^{\varepsilon}\right)} J_{\sharp}^{\varepsilon}(\tilde{\boldsymbol{\Theta}}) .
\end{aligned}
$$

This completes the proof of Theorem 1 .

\section{References}

[1] R.A. Adams, Sobolev Spaces, Academic Press, New York, 1975.

[2] J. Ball, Convexity conditions and existence theorems in nonlinear elasticity, Arch. Rational Mech. Anal. 63 (1977) 337-403.

[3] J. Ball, J. C. Currie, P.J. Olver, Null lagrangians, weak continuity, and variational problems of arbitrary order, J. Funct. Anal. 41 (1981) 135-174. 
[4] R. Bunoiu, P.G. Ciarlet and C. Mardare, Existence theorem for a nonlinear elliptic shell model, J. Elliptic Parabolic Eqs 1 (2015) 31-48.

[5] P. G. Ciarlet, An Introduction to Differential Geometry with Applications to Elasticity, Springer, Dordrecht, 2005.

[6] P. G. Ciarlet and G. Geymonat, Sur les lois de comportement en élasticité non linéaire compressible, C.R. Acad. Sci. Paris, Ser. II, 295 (1982) 423426 .

[7] P.G. Ciarlet, M. Malin and C. Mardare, New nonlinear estimates for surfaces in terms of their fundamental forms, C. R. Acad. Sci. Paris, Ser. I 355 (2017) 226-231.

[8] P.G. Ciarlet and C. Mardare, A mathematical model of Koiter's type for a nonlinearly elastic "almost spherical" shell, C. R. Acad. Sci. Paris, Ser. I 354 (2016) 1241-1247.

[9] P.G. Ciarlet and C. Mardare, A nonlinear shell model of Koiter's type, C. R. Acad. Sci. Paris, Ser. I 356 (2018) 227-234.

[10] G. Friesecke, R. D. James, M. G. Mora and S. Müller, Derivation of nonlinear bending theory for shells from three dimensional nonlinear elasticity by Gamma-convergence, C.R. Acad. Sci. Paris, Sér. I, 336 (2003) 697-702.

[11] F. John, Estimates for the derivatives of the stresses in a thin shell and interior shell equations, Comm. Pure Appl. Math. 18 (1965) 235-267.

[12] F. John, Refined interior equations for thin elastic shells, Comm. Pure Appl. Math. 24 (1971) 583-615.

[13] W. T. Koiter, On the nonlinear theory of thin elastic shells, Proc. Kon. Ned. Akad. Wetensch. B69 (1966) 1-54.

[14] H. Le Dret and A. Raoult, The membrane shell model in nonlinear elasticity: A variational asymptotic derivation, J. Nonlinear Sci. 6 (1996) 59-84.

[15] C. Mardare, On the derivation of nonlinear shell models from threedimensional elasticity, Rev. Roumaine Math. Pures Appl. 53 (2008), 499522 .

[16] J. Nečas, Les Méthodes Directes en Théorie des Équations Elliptiques, Masson, Paris, 1967. English translation: Direct Methods in the Theory of Elliptic Equations, Springer, 2012. 